Article

\title{
On Solving Two-Dimensional Inverse Heat Conduction Problems Using the Multiple Source Meshless Method
}

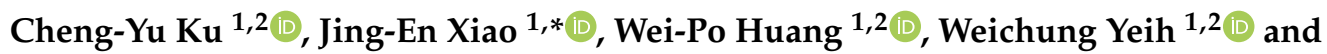 \\ Chih-Yu Liu ${ }^{1}$ (D) \\ 1 Department of Harbor and River Engineering, National Taiwan Ocean University, Keelung 20224, Taiwan \\ 2 Center of Excellence for Ocean Engineering, National Taiwan Ocean University, Keelung 20224, Taiwan \\ * Correspondence: 20452002@email.ntou.edu.tw; Tel.: +886-224-622-192 (ext. 6159)
}

Received: 5 June 2019; Accepted: 27 June 2019; Published: 28 June 2019

Featured Application: The inverse heat conduction problems are often encountered in many engineering fields. In practice, the temperature can be measured only on a portion of the problem boundary. As a result, the information of the remaining boundary is unknown. In this study, we develop the multiple-source meshless method to solve the inverse heat conduction problems. The proposed method can be applied on problems in the doubly-connected domain with remarkably high accuracy, even though the over-specified boundary data are assigned a portion that is less than $1 / 10$ of the overall domain boundary.

\begin{abstract}
In this article, a newly developed multiple-source meshless method (MSMM) capable of solving inverse heat conduction problems in two dimensions is presented. Evolved from the collocation Trefftz method (CTM), the MSMM approximates the solution by using many source points through the addition theorem such that the ill-posedness is greatly reduced. The MSMM has the same superiorities as the CTM, such as the boundary discretization only, and is advantageous for solving inverse problems. Several numerical examples are conducted to validate the accuracy of solving inverse heat conduction problems using boundary conditions with different levels of noise. Moreover, the domain decomposition method is adopted for problems in the doubly-connected domain. The results demonstrate that the proposed method may recover the unknown data with remarkably high accuracy, even though the over-specified boundary data are assigned a portion that is less than $1 / 10$ of the overall domain boundary.
\end{abstract}

Keywords: meshless method; inverse problem; heat conduction problem; domain decomposition method; collocation Trefftz method

\section{Introduction}

The inverse heat conduction problem is the process of computing the unknown temperatures by using over-specified data. For the inverse problem, both Dirichlet and Neumann boundary conditions are over-specified on portions of the boundary and no information on the remaining domain boundary is given. Since the over-specified data are usually adopted from field measurement data, noise from measurement uncertainties is an important factor of the inverse problem. The inverse problem has been comprehensively studied in many fields, including Cauchy problems [1,2], heat conduction analyses [3,4], boundary optimization problems [5], crack identification problems [6], and wave propagation problems [7]. For example, Qian, $\mathrm{Fu}$, and $\mathrm{Li}$ [8] adopted the method of fundamental solutions (MFS) in conjunction with two different regularization methods for solving the Cauchy problem governed by Laplace equation. In addition, Gu et al. [9] used the generalized finite difference 
method (GFDM) to recover the heat source for steady-state heat conduction problems. For the inverse problem, the boundary condition is only given for portions of the domain boundary, and the given boundary data often include different levels of noise in which the problem is usually a severely ill-posed problem. To deal with inverse problems, several numerical techniques have been proposed to improve the ill-conditioning matrix. In 2005, Yeih et al. [10] utilized the MFS in cooperation with the exponentially convergent scalar homotopy algorithm (ECSHA) to treat the ill-posed phenomenon for the inverse Cauchy problem. Bourgeois [11] presented the mixed formulation of quasi-reversibility to solve the Cauchy problem for the Laplace governing equation. In order to use this technique, the numerical results can be carried out easily without using the iterative method. Later, Andrieux, Baranger, and Abda introduced [12] an approach based on the minimization of an energy error functional for temperature and heat flux data recovering. The Tikhonov regularization technique $[13,14]$ is one of the commonly used methods to mitigate the ill-posed phenomenon. Moreover, the L-curve method $[15,16]$ is often found to obtain the regularization parameter. In the past decades, mesh-based numerical methods have been commonly used to solve the inverse problem, such as the finite difference method $[17,18]$ and the boundary element method $[19,20]$. However, the development of new numerical methods has still attracted interests. The meshless approach may be one of the most competitive alternatives recently.

Among the meshless methods, such as the collocation Trefftz method (CTM) [21,22], the MFS [23], boundary knot method [24], element-free Galerkin method [25], radial basis function collocation method [26], multi-scale polynomial expansion method [27], and boundary particle method [28], the boundary-type meshless method is perhaps the most simple and accurate method. The CTM and the MFS are based on the Trefftz method, in which the CTM adopts T-complete functions and the MFS includes the fundamental solution as the basis functions. Since the T-complete functions and the fundamental solution are solutions of the governing equation, the boundary points may only need to be placed on the domain boundary. The CTM and MFS are therefore referred to boundary-type meshless methods, which usually suffer from the numerical instability due to the ill-posed phenomenon of the meshless method. To improve the applicability of the CTM and MFS, Ku et al. [29,30] propose the multiple source meshless method (MSMM). The newly developed MSMM is modified from the CTM and MFS and combines the benefits of both methods. The MSMM resolves the problem of finding proper locations of source points in the MFS and reduces the ill-posed problem from higher order Trefftz terms in the CTM. Consequently, the MSMM can be a promising method for solving inverse heat conduction problems.

In this study, a newly developed MSMM capable of solving inverse heat conduction problems in two dimensions is presented. Based on the CTM, the MSMM approximates the solution by using many source points through the addition theorem. Moreover, the ill-posed phenomenon is considerably more moderated when the MSMM is used compared with when the CTM is used. The MSMM has the same advantages as the CTM such as using only boundary discretization, and is advantageous for solving inverse problems. Several numerical examples are presented to validate the accuracy of solving inverse heat conduction problems. To evaluate the capability of the proposed method, boundary conditions with different levels of noise are assigned. Moreover, the domain decomposition method (DDM) is adopted for problems in a doubly-connected domain. The remainder of this paper is outlined as follows: We introduce the mathematical formulation of the two-dimensional inverse heat conduction problem in Section 2. In Section 3, the detailed numerical implementation of the MSMM is described. Section 4 presents the validation examples. A specific discussion of this article is presented in Section 5. Finally, concluding remarks are provided in Section 6.

\section{Inverse Heat Conduction Problem}

In this study, an inverse heat conduction problem governed by a second-order partial differential equation in two-dimensions is considered. The inverse heat conduction problem is generally ill-posed and numerically unstable. As depicted in Figure 1, both Dirichlet and Neumann boundary conditions 
are imposed on the boundary $\partial \Omega_{1}$, and there is no information on the remaining boundary $\partial \Omega_{2}$. The flowchart of the research for the inverse heat conduction problem in two-dimensions is depicted in Figure 2. It is well known that the inverse heat conduction problem of the heat equation is as follows:

$$
\Delta T=0 \text { in } \Omega
$$

where $T$ denotes the temperature and $\Delta$ represents the Laplacian operator. The Dirichlet, Neumann, and mixed-type boundary conditions are expressed in Equations (2)-(4), respectively:

$$
\begin{gathered}
T(\rho, \theta)=f(\theta), \quad 0 \leq \theta \leq \gamma \pi, \\
T_{n}(\rho, \theta)=g(\theta), \quad 0 \leq \theta \leq \gamma \pi \\
\alpha T(\rho, \theta)+\beta T_{n}(\rho, \theta)=u(\theta), \quad 0 \leq \theta \leq \gamma \pi
\end{gathered}
$$

where $\alpha$ and $\beta$ are constants; $\gamma \leq 2 ; f(\theta), g(\theta)$, and $u(\theta)$ denote unknown functions; and $n$ represents the outward normal direction. Different noise levels are assigned to the boundary condition by using the following equation:

$$
\bar{T}=T\left(1+\frac{s}{100} \times \text { rand }\right)
$$

where $\bar{T}$ denotes noisy data, $s$ represents the noise level, and rand denotes a random number.

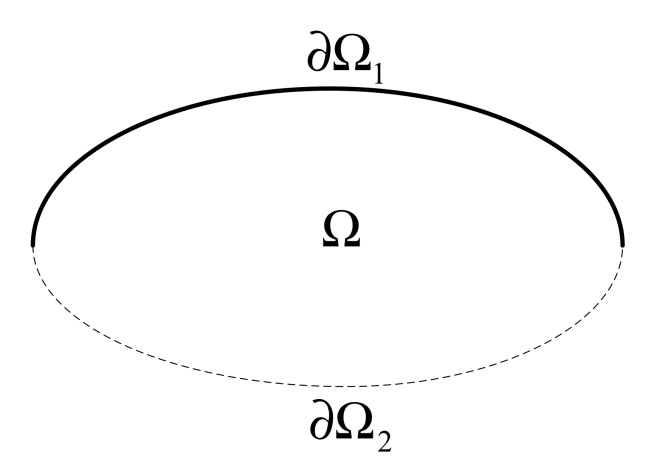

Figure 1. Illustration of the inverse heat conduction problem in two dimensions.

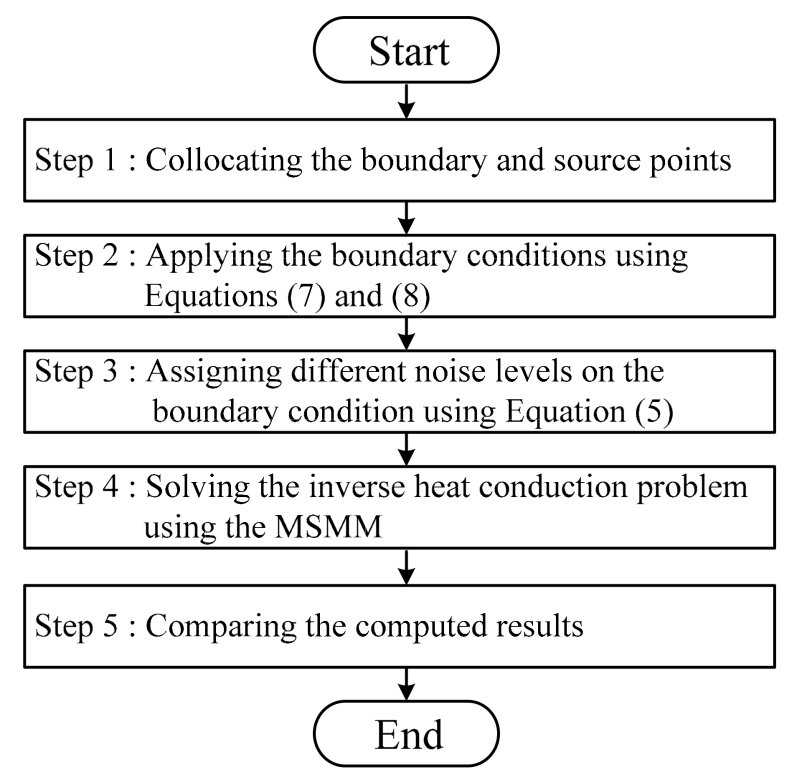

Figure 2. The flowchart of the research. 


\section{Formulation of the Multiple Source Meshless Method}

We may express the heat equation in the dimensionless form as follows:

$$
\frac{\partial^{2} T}{\partial \rho^{2}}+\frac{1}{\rho} \frac{\partial^{2} T}{\partial \rho^{2}}+\frac{1}{\rho^{2}} \frac{\partial^{2} T}{\partial \theta^{2}}=0
$$

where $\rho=\hat{\rho} / R_{0}$. The radial coordinate is denoted by $\rho$ and the angular coordinate is denoted by $\theta$. Figure 3 displays the point distributions for the CTM, MFS, and MSMM in the simply-, doubly-, and multiply-connected regions. By using the additional theorem, approximation solutions can be obtained through the linear superposition principle of the particular solutions by arranging multiple sources within the domain, as shown in Figure 4 . Then, the unknown coefficients $a_{\mathrm{jk}}$ and $b_{\mathrm{jk}}$ are determined using the boundary values as follows:

$$
\begin{gathered}
T(\rho, \theta) \approx \sum_{j=1}^{N_{P}} \sum_{k=1}^{N_{q}}\left[a_{\mathrm{jk}}\left(\rho / R_{0}\right)^{k} \cos k \theta+b_{\mathrm{jk}}\left(\rho / R_{0}\right)^{k} \sin k \theta\right], \\
\frac{\partial T(\rho, \theta)}{\partial n} \approx \sum_{j=1}^{N_{p}} \sum_{k=1}^{N_{q}}\left[a_{\mathrm{jk}} \frac{\partial\left(\left(\rho / R_{0}\right)^{k} \cos k \theta\right)}{\partial n}+b_{\mathrm{jk}} \frac{\partial\left(\left(\rho / R_{0}\right)^{k} \sin k \theta\right)}{\partial n}\right],
\end{gathered}
$$

where $a_{\mathrm{jk}}$ and $b_{\mathrm{jk}}$ denote unknown coefficients, $N_{p}$ denotes the source point number, and $N_{q}$ denotes the number of Trefftz terms. The characteristic length $R_{0}$ is adopted from [31] as:

$$
R_{0}=\xi \times \max (\rho),
$$

where $\xi$ denotes a constant that is a user-defined parameter and $\max (\rho)$ represents the maximum distance between the boundary and source points of the problem. By substituting Equations (7) and (8) into Equations (2) and (3), we obtain the Dirichlet and Neumann boundary conditions as follows:

$$
\begin{aligned}
& T(\rho, \theta) \approx \sum_{j=1}^{N_{p}} \sum_{k=1}^{N_{q}}\left[a_{j k} C_{k}+b_{j k} D_{k}\right], \\
& \frac{\partial T(\rho, \theta)}{\partial n} \approx \sum_{j=1}^{N_{p}} \sum_{k=1}^{N_{q}}\left[a_{j k} E_{k}+b_{j k} F_{k}\right],
\end{aligned}
$$

where $C_{k}:=\left(\frac{\rho}{R_{0}}\right)^{k} \cos k \theta, D_{k}:=\left(\frac{\rho}{R_{0}}\right)^{k} \sin k \theta, E_{k}:=\frac{\partial\left(\left(\frac{\rho}{R_{0}}\right)^{k} \cos k \theta\right)}{\partial n}$, and $F_{k}:=\frac{\partial\left(\left(\frac{\rho}{R_{0}}\right)^{k} \sin k \theta\right)}{\partial n}$.

By using Equations (10) and (11), we obtain a linear equation system as follows:

$$
A \alpha=b .
$$

Then, Equation (12) can be rewritten as follows:

$$
A=\left[\begin{array}{ccccc}
D_{1, k=1}^{I} & D_{1, k=1}^{I I} & \ldots & D_{1, k=N q}^{I} & D_{1, k=N q}^{I I} \\
D_{2, k=1}^{I} & D_{2, k=1}^{I I} & \ldots & D_{2, k=N q}^{I} & D_{2, k=N q}^{I I} \\
D_{3, k=1}^{I} & D_{3, k=1}^{I I} & \ldots & D_{3, k=N q}^{I} & D_{3, k=N q}^{I I} \\
\vdots & \vdots & \ldots & \vdots & \vdots \\
D_{j, k=1}^{I} & D_{j, k=1}^{I I} & \ldots & D_{j, k=N q}^{I} & D_{j, k=N q}^{I I} \\
N_{1, k=1}^{I} & N_{1, k=1}^{I I} & \ldots & N_{1, k=N q}^{I} & N_{1, k=N q}^{I I} \\
N_{2, k=1}^{I} & N_{2, k=1}^{I I} & \ldots & N_{2, k=N q}^{I} & N_{2, k=N q}^{I I} \\
N_{3, k=1}^{I} & N_{3, k=1}^{I I} & \ldots & N_{3, k=N q}^{I} & N_{3, k=N q}^{I I} \\
\vdots & \vdots & \ldots & \vdots & \vdots \\
N_{j, k=1}^{I} & N_{j, k=1}^{I I} & \ldots & N_{j, k=N q}^{I} & N_{j, k=N q}^{I I}
\end{array}\right], \alpha=\left[\begin{array}{c}
\alpha_{0} \\
\alpha_{1} \\
\alpha_{2} \\
\alpha_{3} \\
\vdots \\
\vdots \\
\vdots \\
\alpha_{N q}-2 \\
\alpha_{N q}-1 \\
\alpha_{N q}
\end{array}\right], b=\left[\begin{array}{c}
g_{1} \\
g_{2} \\
g_{3} \\
\vdots \\
g_{i} \\
f_{1} \\
f_{2} \\
f_{3} \\
\vdots \\
f_{j}
\end{array}\right],
$$

where $D_{j, k}^{I}:=\left(\rho_{\mathrm{j}} / \mathrm{R}_{0}\right)^{\mathrm{k}} \cos \left(\mathrm{k} \theta_{\mathrm{j}}\right) ; D_{j, k}^{I I}:=\left(\rho_{\mathrm{j}} / \mathrm{R}_{0}\right)^{\mathrm{k}} \sin \left(\mathrm{k} \theta_{\mathrm{j}}\right)$; 


$$
\begin{aligned}
N_{j, k}^{I}:=\begin{array}{c}
{\left[\left(k\left(1 / R_{0}\right)^{k} \rho_{j}^{k-1} \cos \left(k \theta_{j}\right) \cos \theta_{j}\right)+\left(k\left(1 / R_{0}\right)^{k} \rho_{j}^{k-1} \sin \left(k \theta_{j}\right) \sin \theta_{j}\right)\right] n_{x}} \\
+\left[\left(k\left(1 / R_{0}\right)^{k} \rho_{j}^{k-1} \cos \left(k \theta_{j}\right) \sin \theta_{j}\right)-\left(k\left(1 / R_{0}\right)^{k} \rho_{j}^{k-1} \sin \left(k \theta_{j}\right) \cos \theta_{j}\right)\right] n_{y}
\end{array} ; \text { and } \\
N_{j, k}^{I I}:=\begin{array}{l}
{\left[\left(k\left(1 / R_{0}\right)^{k} \rho_{j}^{k-1} \sin \left(k \theta_{j}\right) \cos \theta_{j}\right)-\left(k\left(1 / R_{0}\right)^{k} \rho_{j}^{k-1} \cos \left(k \theta_{j}\right) \sin \theta_{j}\right)\right] n_{x}} \\
+\left[\left(k\left(1 / R_{0}\right)^{k} \rho_{j}^{k-1} \sin \left(k \theta_{j}\right) \sin \theta_{j}\right)+\left(k\left(1 / R_{0}\right)^{k} \rho_{j}^{k-1} \cos \left(k \theta_{j}\right) \cos \theta_{j}\right)\right] n_{y}
\end{array} .
\end{aligned}
$$

Here, $A$ denotes the coefficient matrix, $\alpha$ denotes the unknown coefficients and is given as $\alpha=\left[a_{0}, \ldots, N_{q}\right]^{T}$, and $b$ denotes the function value from the boundary conditions. The DDM [32] is adopted for problems in the doubly-connected domain. An epitrochoid-shaped doubly-connected domain is depicted in Figure 5. We split the domain into $\Omega_{1}$ and $\Omega_{2}$. The sub-boundaries include $\Gamma_{1}$, $\Gamma_{2}, \ldots$, and $\Gamma_{8} . \Omega_{1}$ comprises $\Gamma_{1}, \Gamma_{2}, \Gamma_{3}$, and $\Gamma_{4}$, and $\Omega_{2}$ comprises $\Gamma_{5}, \Gamma_{6}, \Gamma_{7}$, and $\Gamma_{8}$. The interface must satisfy the following conditions:

$$
\left.T\right|_{\Gamma_{1}}=\left.T\right|_{\Gamma_{5}},\left.\quad \frac{\partial T}{\partial n}\right|_{\Gamma_{1}}=\left.\frac{\partial T}{\partial n}\right|_{\Gamma_{5}},\left.\quad T\right|_{\Gamma_{3}}=\left.T\right|_{\Gamma_{7}},\left.\quad \frac{\partial T}{\partial n}\right|_{\Gamma_{3}}=\left.\frac{\partial T}{\partial n}\right|_{\Gamma_{7}} .
$$

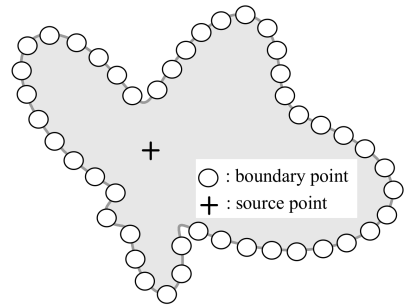

Simply-connected domain
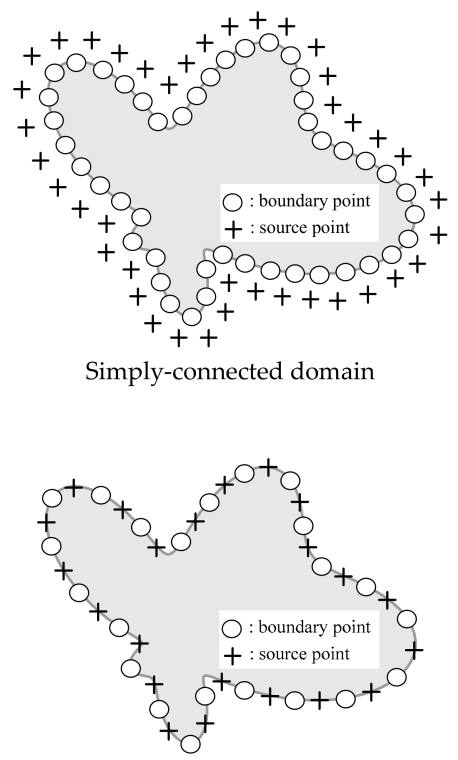

Simply-connected domain

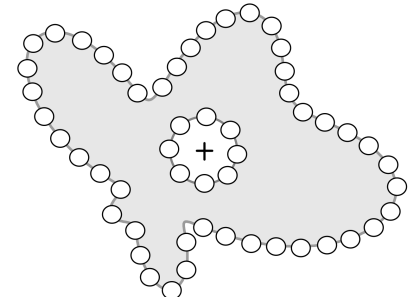

Doubly-connected domain

(a)

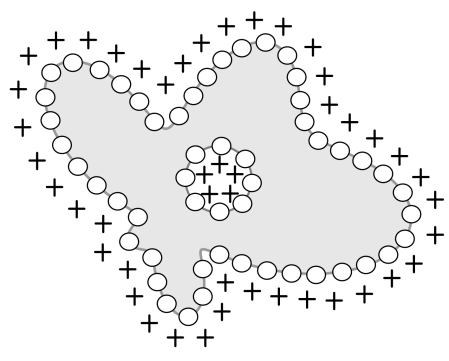

Doubly-connected domain

(b)

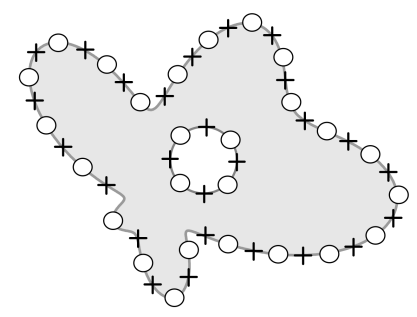

Doubly-connected domain

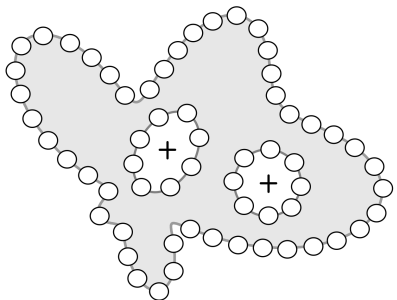

Multiply-connected domain

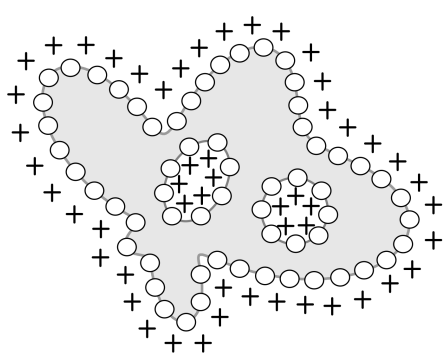

Multiply-connected domain

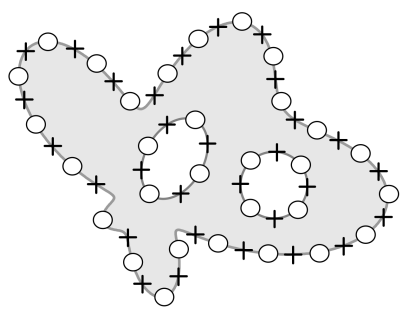

Multiply-connected domain

(c)

Figure 3. Illustration of the collocation points for the collocation Trefftz method, method of fundamental solutions, and multiple source meshless method. (a) Collocation scheme for the CTM, (b) Collocation scheme for the MFS, (c) Collocation scheme for the MSMM.

From this equation, Equation (14) is rewritten as follows:

$$
\frac{\partial T(x)}{\partial n}=\frac{\partial T(x)}{\partial x} n_{x}+\frac{\partial T(x)}{\partial y} n_{y}
$$


where $n_{x}$ and $n_{y}$ represent the outward normal direction in the $x$ and $y$ direction, respectively. The final expression for determining the unknown coefficients $\alpha_{D}$ can be expressed as follows:

$$
\begin{gathered}
A_{D} \alpha_{D}=b_{D} \\
A_{D}=\left[\begin{array}{cc}
A_{\Omega_{1}} & 0_{\Omega_{2}} \\
\left.A_{I}\right|_{\Gamma_{1}, \Gamma_{3}} & \left.A_{I}\right|_{\Gamma_{5}, \Gamma_{7}} \\
0_{\Omega_{1}} & A_{\Omega_{2}}
\end{array}\right], \quad \alpha_{D}=\left[\begin{array}{c}
\alpha_{\Omega_{1}} \\
\alpha_{\Omega_{2}}
\end{array}\right], \quad b_{D}=\left[\begin{array}{c}
b_{\Omega_{1}} \\
b_{I} \\
b_{\Omega_{2}}
\end{array}\right]
\end{gathered}
$$

where $A_{\Omega_{1}}$ and $A_{\Omega_{2}}$ denote the coefficient matrix of $\Omega_{1}$ and $\Omega_{2}$, respectively. $\left.A_{I}\right|_{\Gamma_{1}, \Gamma_{3}}$ and $\left.A_{I}\right|_{\Gamma_{5}, \Gamma_{7}}$ represent the matrices for the boundary $\Gamma_{1}, \Gamma_{3}, \Gamma_{5}$, and $\Gamma_{7}$ at the interface. $0_{\Omega_{1}}$ and $0_{\Omega_{2}}$ represent a zero matrix, $\alpha_{\Omega_{1}}$ and $\alpha_{\Omega_{2}}$ are the vectors of unknown coefficients, $b_{I}=[0]$ represents the boundary values imposed on the collocation points at the interface, and 0 denotes zero vectors. By solving the linear equation system of the form presented in Equation (16), unknown coefficients, $\alpha_{\Omega_{1}}$ and $\alpha_{\Omega_{2}}$ for $\Omega_{1}$ and $\Omega_{2}$, respectively, can then be determined.

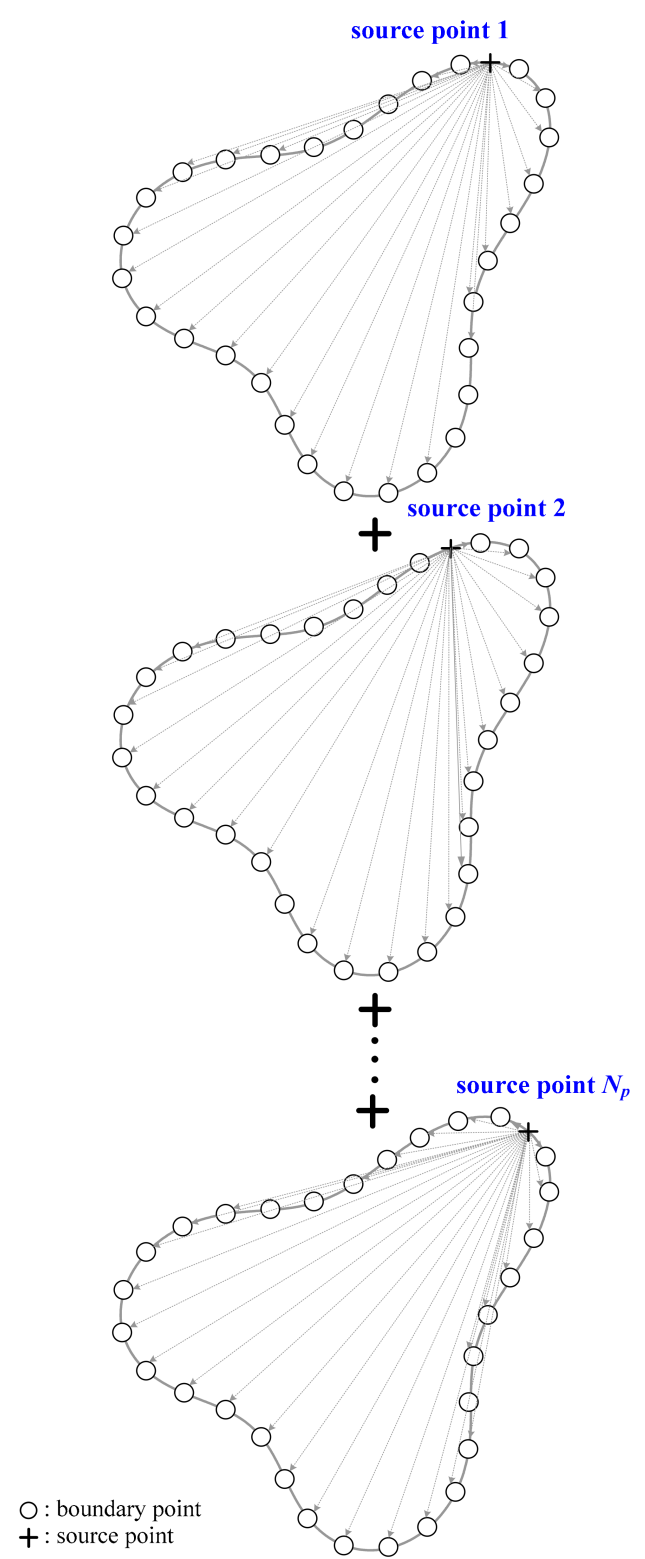

Figure 4. Illustration of the addition theorem for the multiple source meshless method. 

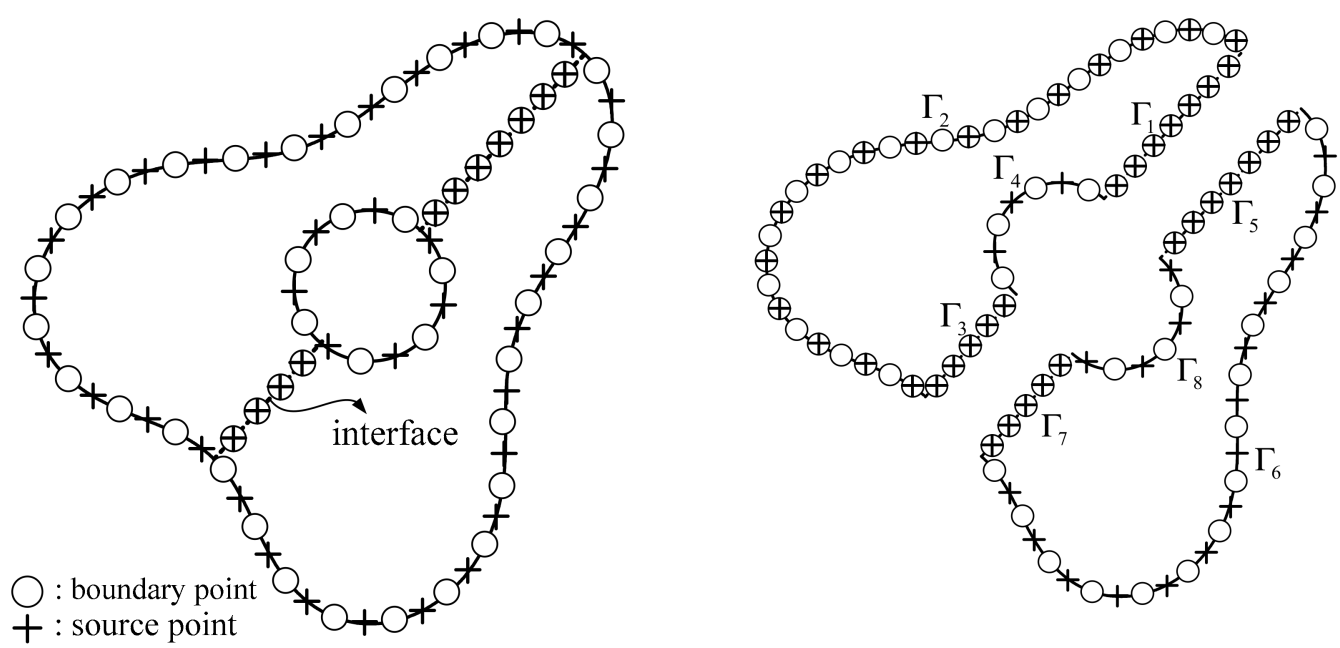

Figure 5. Illustration of the domain decomposition method (DDM) for a doubly-connected domain.

\section{Validation of the Proposed Approach}

\subsection{Example 1}

This example is used to validate the two-dimensional inverse heat conduction problem in a simply connected domain that is bounded by an amoeba-shaped section, as depicted in Figure 6. We verify the accuracy of the proposed method, and the numerical results of the proposed method are compared with those of the MFS. The domain boundary is described as follows:

$$
\Gamma=\{(x, y) \mid x=\rho \cos \theta, y=\rho \sin \theta\}, \quad \rho=e^{(\sin \theta \sin 2 \theta)^{2}}+e^{(\cos \theta \cos 2 \theta)^{2}}, \quad 0 \leq \theta \leq 2 \pi
$$

where the radial coordinate is denoted by $\rho$ and the angular coordinate is denoted by $\theta$. The analytical solution is given by:

$$
T=e^{x} \cos y+e^{x} \sin y .
$$

In this study, we assign the boundary data to the domain boundary in the range of $0 \leq \theta \leq 1.5 \pi$, and there is no information on the remaining boundary. The boundary conditions including $\Gamma_{1}$ and $\Gamma_{2}$ are displayed in Figure 6. At $\Gamma_{1}$, the over-specified Dirichlet and Neumann boundary conditions are given on the boundary collocation points by using the analytical solution, as presented in Equation (19).

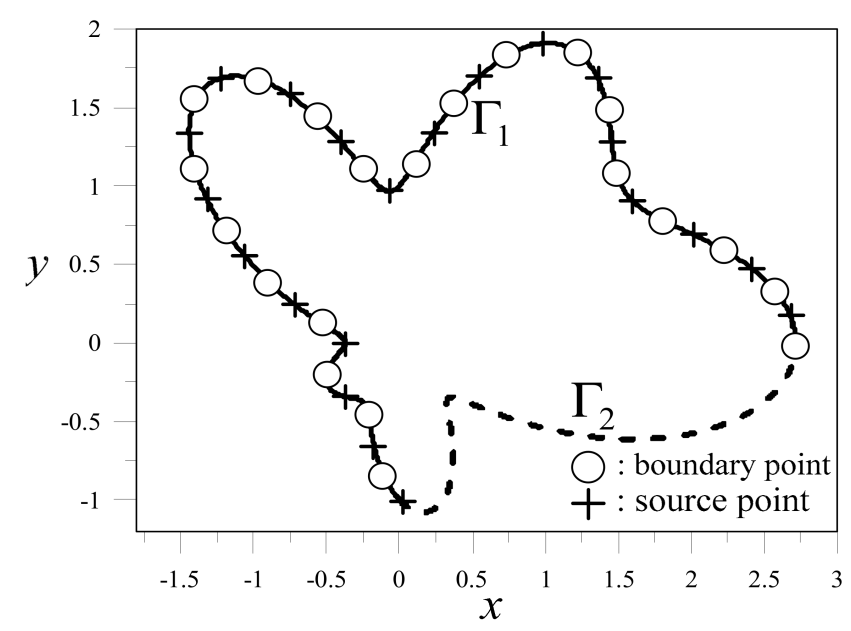

Figure 6. Illustration of the collocation points and boundary conditions. 
In this example, the parameter $N_{q}$ represents the number of terms for the non-singular basis functions and is set as 10 . We first investigated the accuracy of the proposed method by selecting the terms of the Trefftz order through different $N_{q}$ values, as depicted in Figure 7. The results demonstrate that the accuracy of the proposed method can reach the order of $10^{-7}$ when $N_{q}=20$. Furthermore, the results of the maximum absolute error (MAE) versus the source point number are presented in Figure 8. By using $N_{q}=20$, we can obtain an excellent accuracy with an MAE of $10^{-7}$ while locating more than 50 source points on the domain. In this example, we arrange 58 points inside the domain to approximate the temperature field. The contour of the temperature is shown in Figure 9. The computed temperature along line $\mathrm{AB}$ is revealed to be almost identical to the exact solution, as depicted in Figure 10. Moreover, the MFS is adopted to solve the same problem and is compared with the proposed method. The results demonstrate that the MAE of the MFS in example 1 is approximately $10^{-3}$.

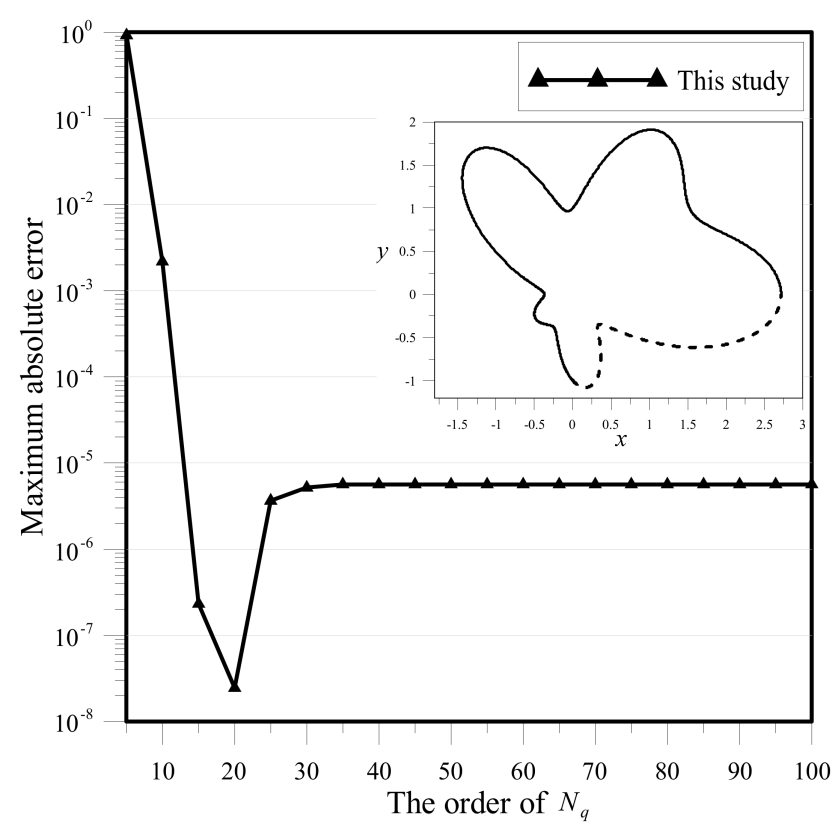

Figure 7. $N_{q}$ versus the maximum absolute error.

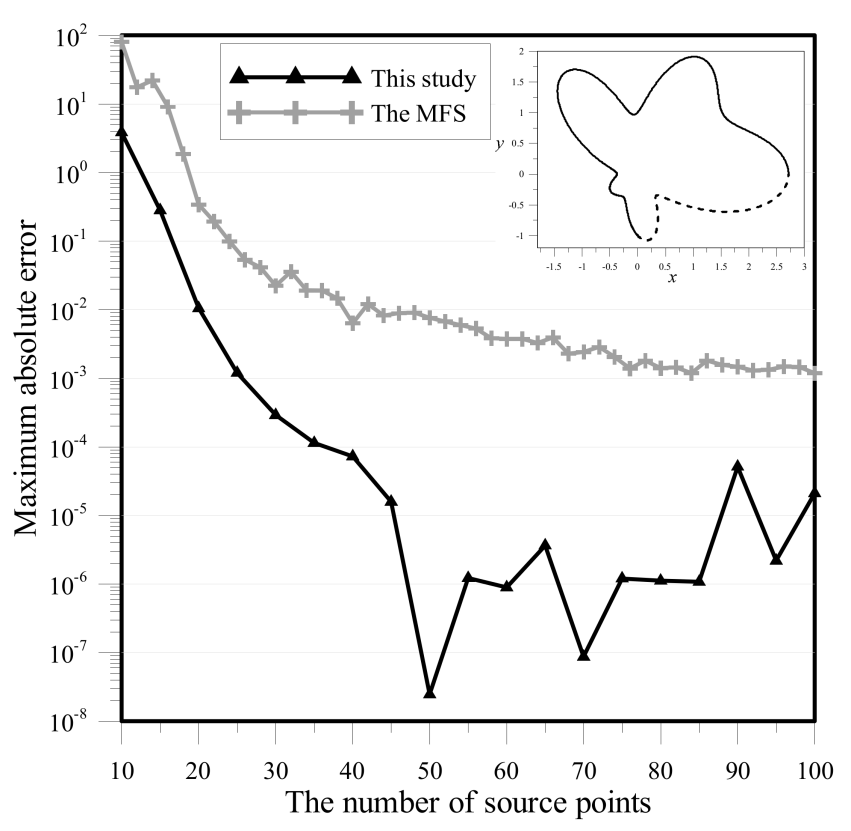

Figure 8. Source point number versus the MAE. 


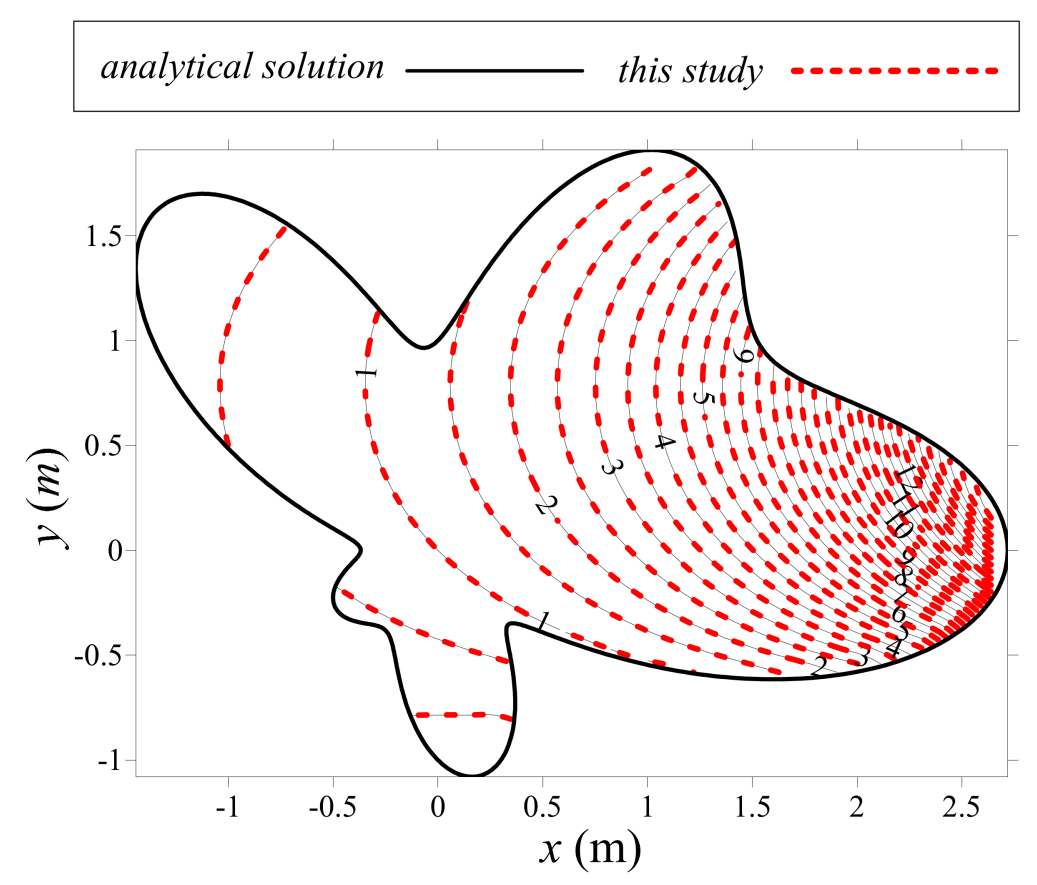

Figure 9. Comparison of the numerical solution with the exact solution.

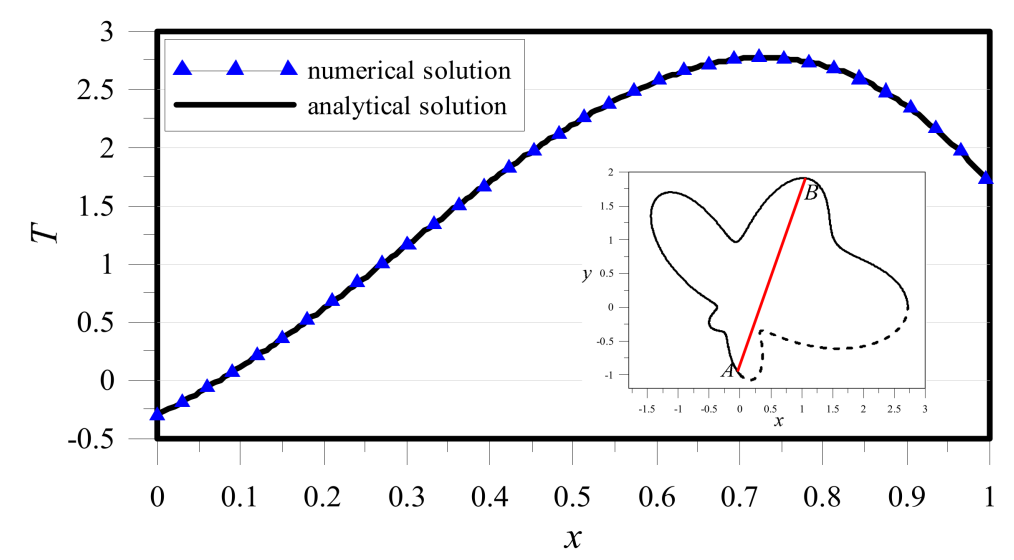

Figure 10. Comparison of the temperature distribution along the selected line AB.

\subsection{Example 2}

We investigate a two-dimensional inverse heat conduction problem in a star-shaped simply connected domain, as depicted in Figure 11. The two-dimensional object boundary is described by the following equation:

$$
\Gamma=\{(x, y) \mid x=\rho \cos \theta, y=\rho \sin \theta\}, \quad \rho=1+\cos ^{2}(4 \theta), 0 \leq \theta \leq 2 \pi,
$$

where the radial coordinate is denoted by $\rho$ and the angular coordinate is denoted by $\theta$. The analytical solution is given by:

$$
T=x^{2}-y^{2}
$$

The parameter $N_{q}$ represents the number of terms in the non-singular basis functions and is set as 10. Figure 11 displays the distribution of the collocation points on the domain boundary. The Dirichlet and Neumann boundary conditions are given for a portion of the domain boundary by designating the exact solution as expressed in Equation (21). The MFS is also adopted to solve the same problem. 


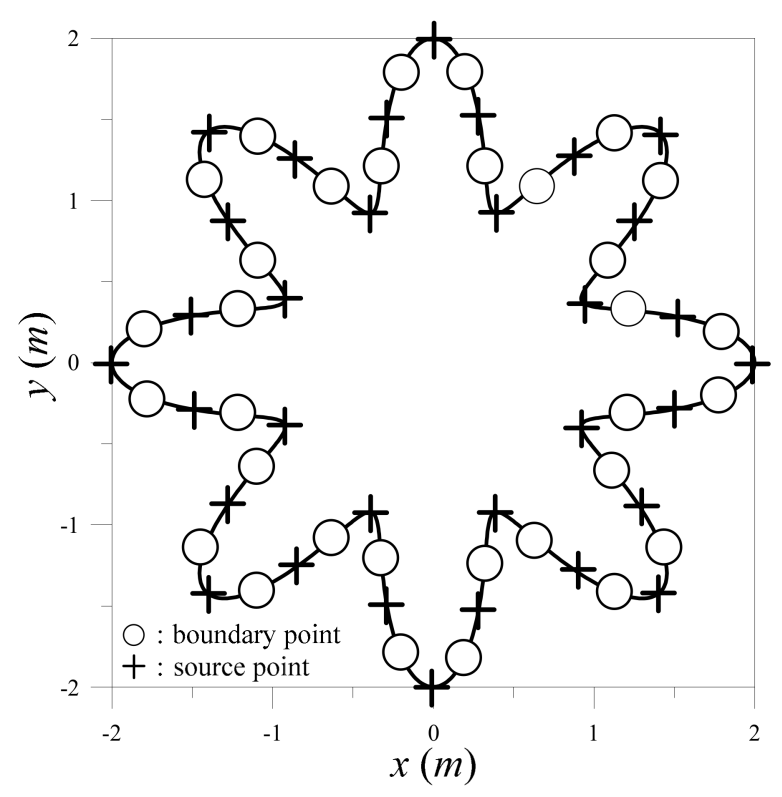

Figure 11. Boundary collocation points.

In this example, we define a parameter $\zeta$, which presents the boundary data coverage. If $\zeta=2 \pi$, then the boundary conditions are given for the whole domain boundary. This causes the problem to become a direct problem. By contrast, for the inverse problem, the boundary conditions are given only for a portion of the domain boundary if $\zeta<2 \pi$.

Figure 12 presents the accuracy of the computed results of $\zeta$ versus those of the MAE. The results reveal that the accuracy of the MSMM is superior to that of the MFS. We collocate 200 inner points within the physical boundary to obtain the temperature field of the problem, as shown in Figure 13. The numerical results reveal that the proposed method can recover the unknown data with a high level of accuracy, although the over-specified data are provided a portion that is less than $1 / 10$ of the overall boundary.

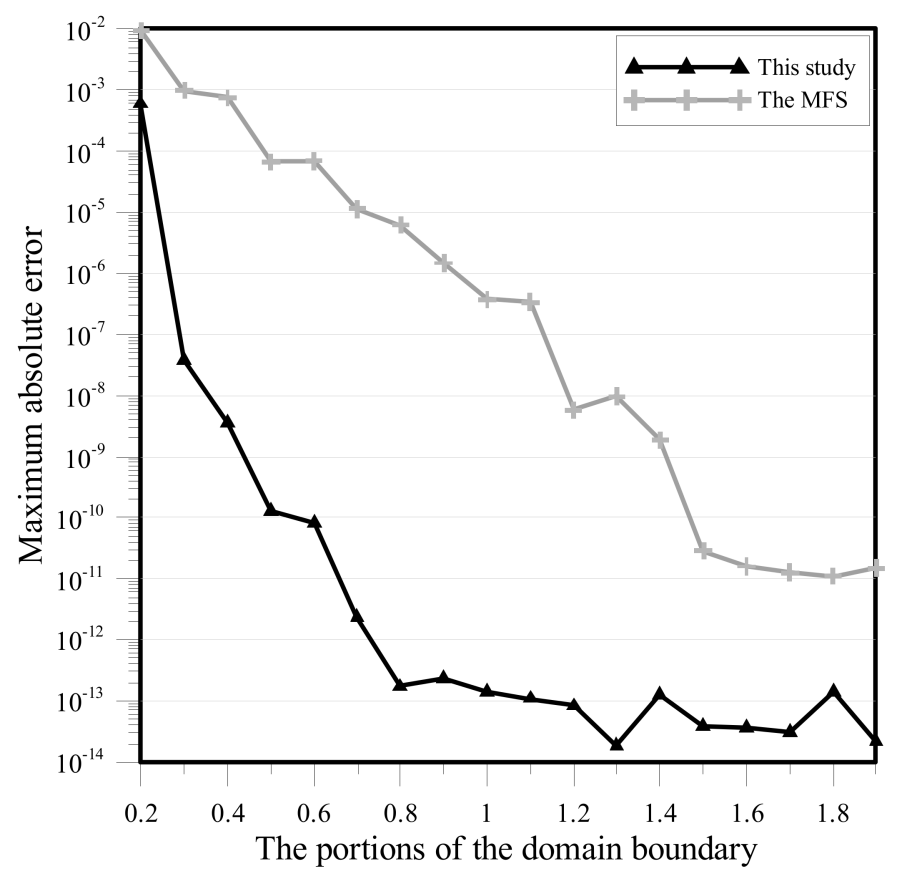

Figure 12. $\zeta$ versus the MAE. 


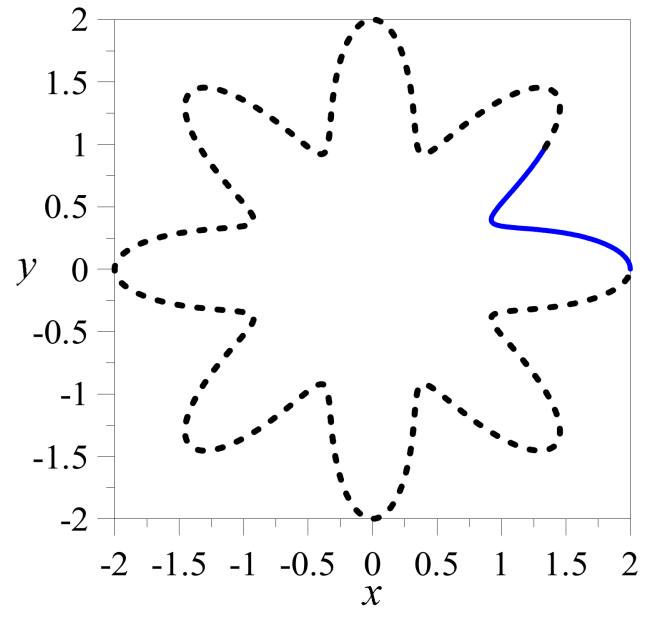

(a)

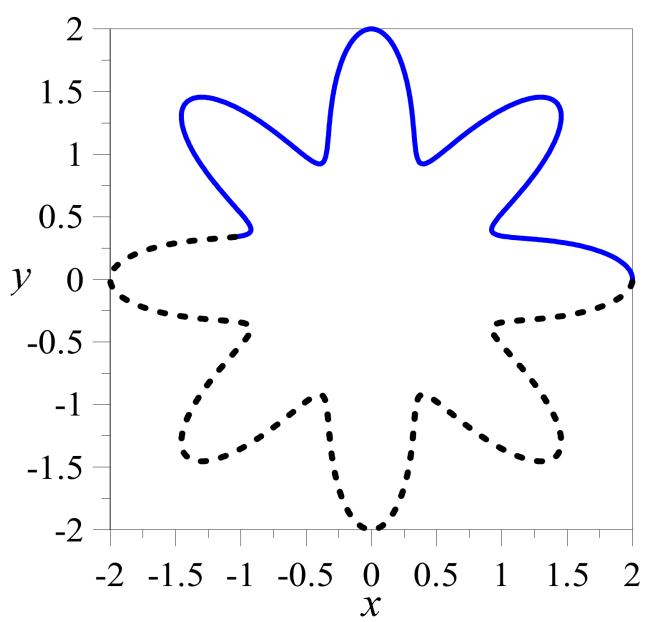

(c)

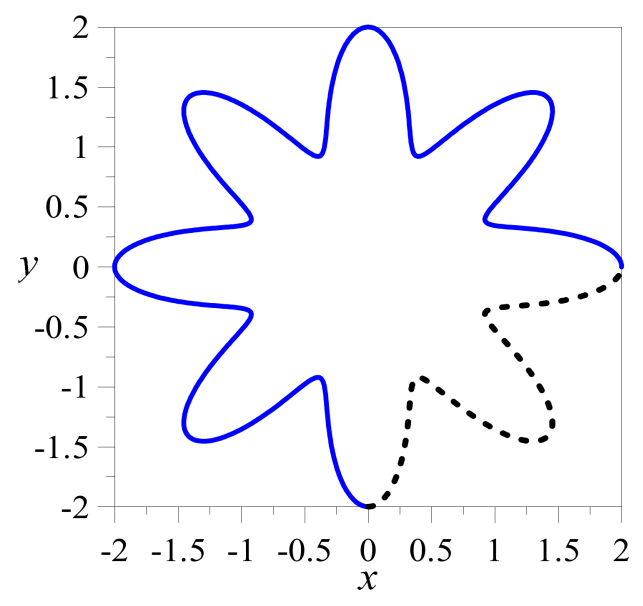

(e)

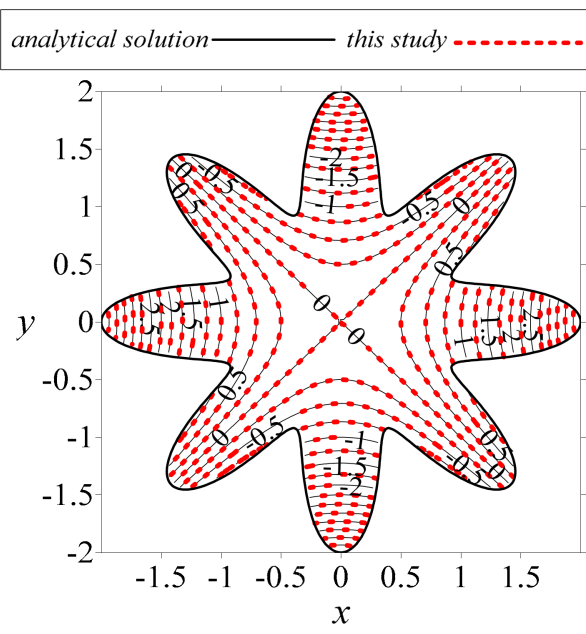

(b)

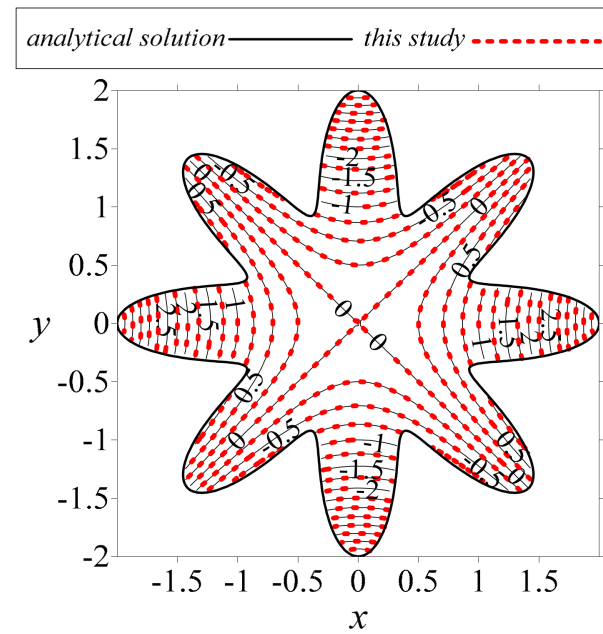

(d)

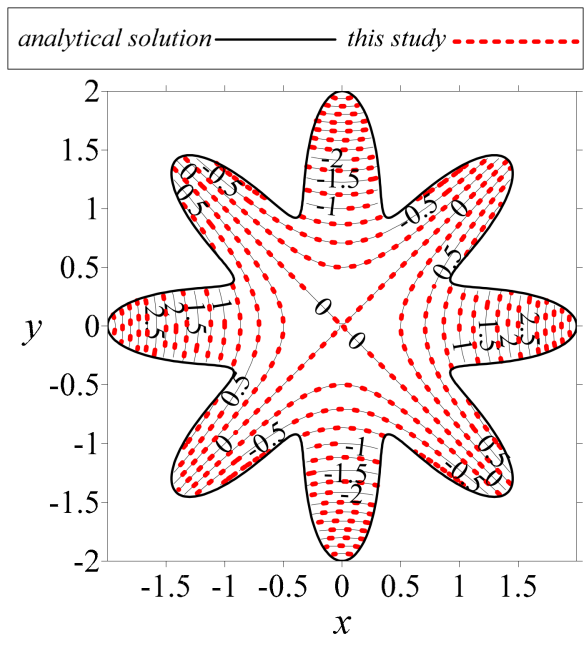

(f)

Figure 13. Comparison of the temperature fields of the multiple source meshless method. (a,c,e) Over-specified boundary conditions are given on the 2/20,9/20, and 15/20 portion of the overall boundary, (b) results comparison with boundary data of $\theta$ values in the range of $0 \leq \theta \leq 0.2 \pi$, (d) results comparison with boundary data of $\theta$ values in the range of $0 \leq \theta \leq 0.9 \pi$, and (f) results comparison with boundary data of $\theta$ values in the range of $0 \leq \theta \leq 1.5 \pi$ 


\subsection{Example 3}

In this example, we investigate a two-dimensional inverse heat conduction problem in a peanut-shaped simply connected domain, as depicted in Figure 14. To evaluate the accuracy of the MSMM, we compare the computed results of the proposed method and those of the MFS used with the ECSHA [10]. The two-dimensional object boundary is described by the following equation:

$$
\Gamma=\{(x, y) \mid x=\rho \cos \theta, y=\rho \sin \theta\}, \quad \rho=\sqrt{\cos (2 \theta)+\sqrt{1.1-\sin ^{2}(2 \theta)}}, \quad 0 \leq \theta \leq 2 \pi
$$

where the radial coordinate is denoted by $\rho$ and the angular coordinate is denoted by $\theta$, respectively. The analytical solution is given by:

$$
T=e^{x} \cos y .
$$

In this study, the parameter $N_{q}$ denotes the number of terms of the non-singular basis functions and is set as 10. Figure 14 shows the distribution of the collocation points on the domain boundary. In total, 100 boundary and source points are uniformly placed on the domain boundary. Two scenarios are considered in this example. Figure 15a depicts the temperature distribution of the exact solution. For the first scenario, the boundary data are imposed on the $\theta$ values in the range of $0 \leq \theta \leq 3 / 2 \pi$ with noise levels of $s=0$ and $s=5$. We collocate 200 inner points within the domain boundary. The temperature distributions are illustrated in Figure 15c,e. The MFS has been used to obtain the numerical results, as depicted in Figure 15b,d. For the second scenario, the boundary data are imposed on the $\theta$ values in the range of $0 \leq \theta \leq \pi$ with noise levels of $s=0$ and $s=5$. We collocate 200 points inside the domain to obtain the temperature field of the problem, as shown in Figure 15g,i. The MFS has been also used to obtain the numerical results, as depicted in Figure 15f,h. The MAE by assigning different levels of noise for example 3 is depicted in Table 1. To show the accuracy of the MSMM, we consider that the boundary conditions are polluted by random noise where the noise level is $s=5$. It is found that the numerical solution of the temperature field can be approximated in a stable way, and the accuracy of the numerical solution decreases with the increasing noise level. The computed results demonstrate that the accuracy of the proposed method can reach to the order of $10^{-2}$ while $s=5$. The accuracy level of the MSMM is discovered to be higher than that of the MFS used with the ECSHA when $s=5$.

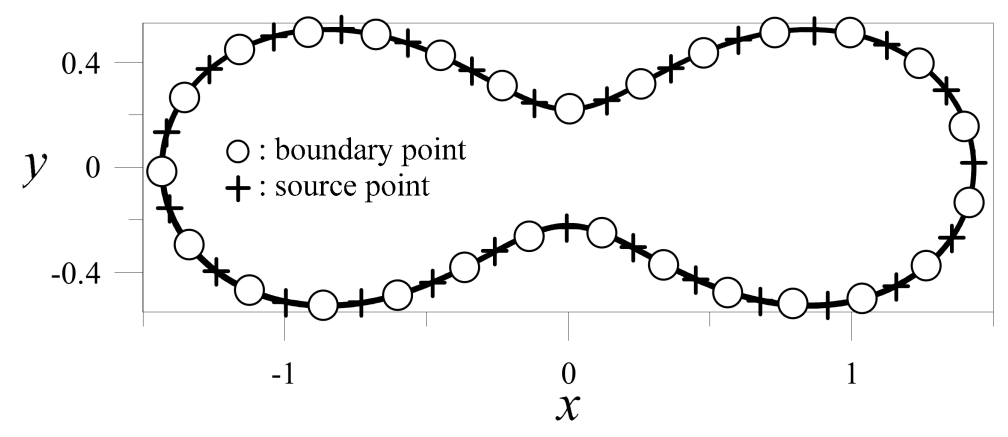

Figure 14. Boundary collocation points.

Table 1. The MAE by assigning different levels of noise for example 3.

\begin{tabular}{ccc}
\hline Boundary Data & Noise Level & Maximum Absolute Error \\
\hline $0 \leq \theta \leq 3 / 2 \pi$ & $s=0$ & $8.43 \times 10^{-6}$ \\
$0 \leq \theta \leq 3 / 2 \pi$ & $s=5$ & $7.12 \times 10^{-2}$ \\
$0 \leq \theta \leq \pi$ & $s=0$ & $3.32 \times 10^{-4}$ \\
$0 \leq \theta \leq \pi$ & $s=5$ & $8.66 \times 10^{-1}$ \\
\hline
\end{tabular}




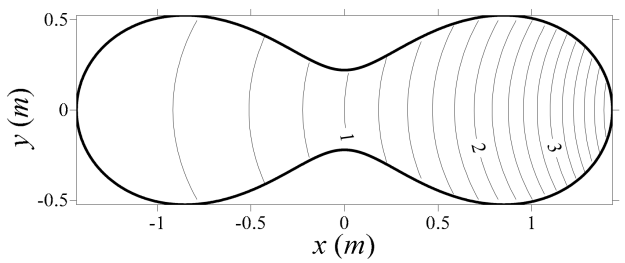

(a)

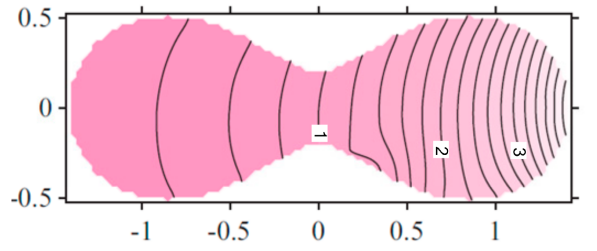

(b)

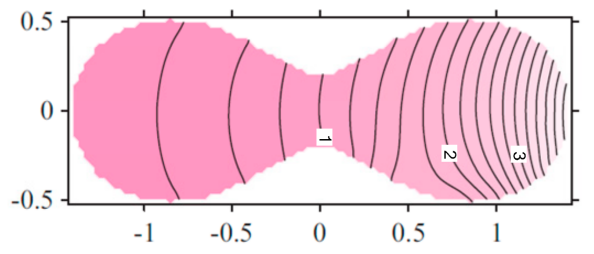

(d)

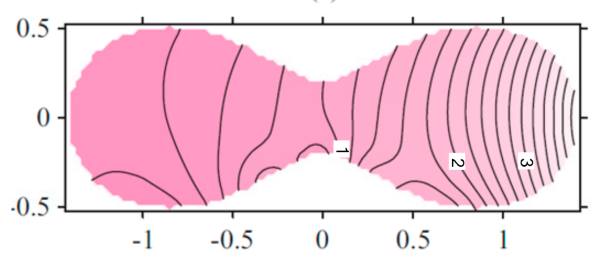

(f)

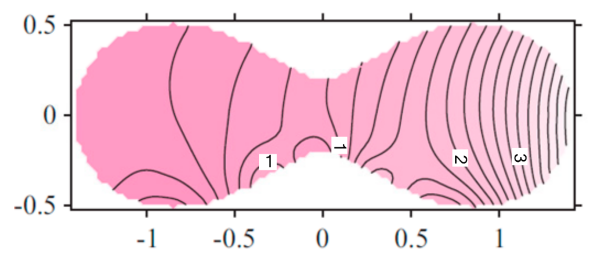

(h)

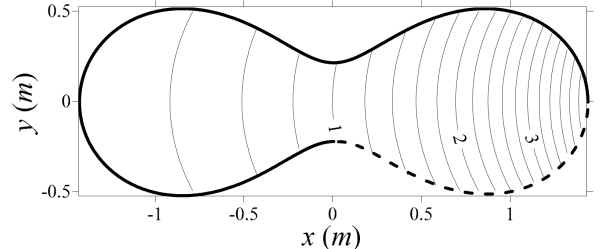

(c)

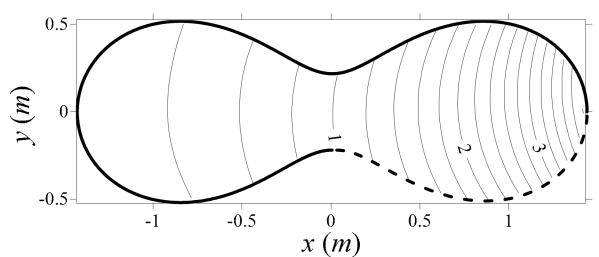

(e)

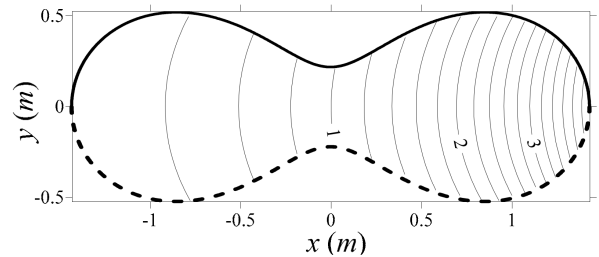

(g)

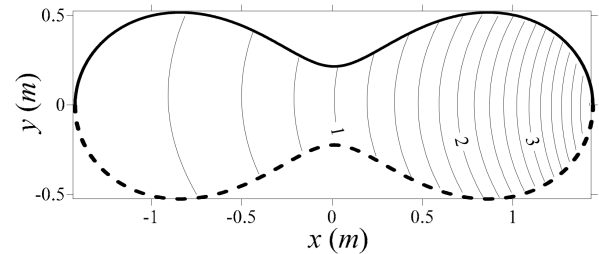

(i)

Figure 15. Comparison of the temperature fields of the proposed method and the MFS. (a) Analytical solution, $(\mathbf{b}, \mathbf{c})$ results with boundary data of $\theta$ values in the range of $0 \leq \theta \leq 3 / 2 \pi$ with a noise level of $s=0,(\mathbf{d}, \mathbf{e})$ the results with boundary data of $\theta$ values in the range of $0 \leq \theta \leq 3 / 2 \pi$ with a noise level of $s=5,(\mathbf{f}, \mathbf{g})$ the results with boundary data of $\theta$ values in the range of $0 \leq \theta \leq \pi$ with a noise level of $s=0$, and $(\mathbf{h}, \mathbf{i})$ the results with boundary data of $\theta$ values in the range of $0 \leq \theta \leq \pi$ with a noise level of $s=5$.

\subsection{Example 4}

In this example, we investigate a two-dimensional inverse heat conduction problem in a rectangular simply connected domain, as depicted in Figure 16. To evaluate the accuracy of the MSMM, we compare the computed results of this method with those of the weighted reproducing kernel collocation method (weighted RKCM) [33]. The two-dimensional object boundary is described by the following equation:

$$
\Gamma=\{(x, y) \mid 0 \leq x \leq 1,0 \leq y \leq 1\},
$$


where the radial coordinate is denoted by $\rho$ and the angular coordinate is denoted by $\theta$. The analytical solution is given by:

$$
T=-x^{3}-y^{3}+3 x y^{2}+3 x^{2} y
$$

$N_{q}$ represents the number of terms in the non-singular basis functions and is set as be 10 . We give the boundary data on the $\Gamma_{2}, \Gamma_{3}$, and $\Gamma_{4}$, and there is no information on the $\Gamma_{1}$. Figure 16 shows the distribution of the collocation points arranged in the domain. In this example, 400 collocation points are placed. The boundary data are imposed on $\Gamma_{2}, \Gamma_{3}$, and $\Gamma_{4}$ with noise levels of $s=0, s=1$, and $s=2$. We collocate 200 inner points to obtain the temperature field of the problem, as shown in Figure 17. Figure 18 illustrates a comparison of the absolute error of the proposed method and the weighted RKCM. To evaluate the stability of the MSMM, different noise levels of $s=0, s=1$, and $s=2$ were considered in the numerical model. The MAE by assigning different levels of noise for example 4 is depicted in Table 2. It can be seen that the absolute error is relatively small, and the accuracy of the numerical solution decreases with the increasing noise level. The computed results demonstrate that the accuracy of the proposed method can reach to the order of $10^{-3}$ while $s=2$ is considered. From Figure 19, we discover that the results of the proposed method for $\Gamma_{1}$ are almost identical with the exact solution.

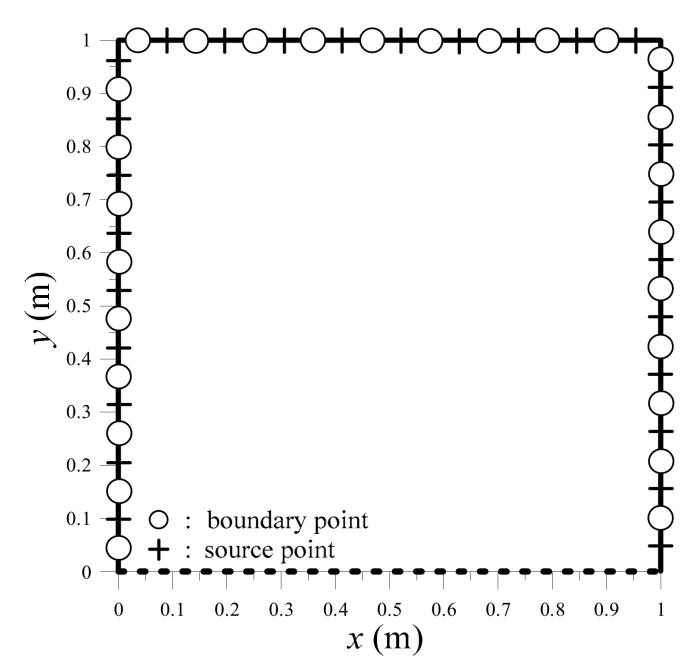

Figure 16. Boundary collocation points.

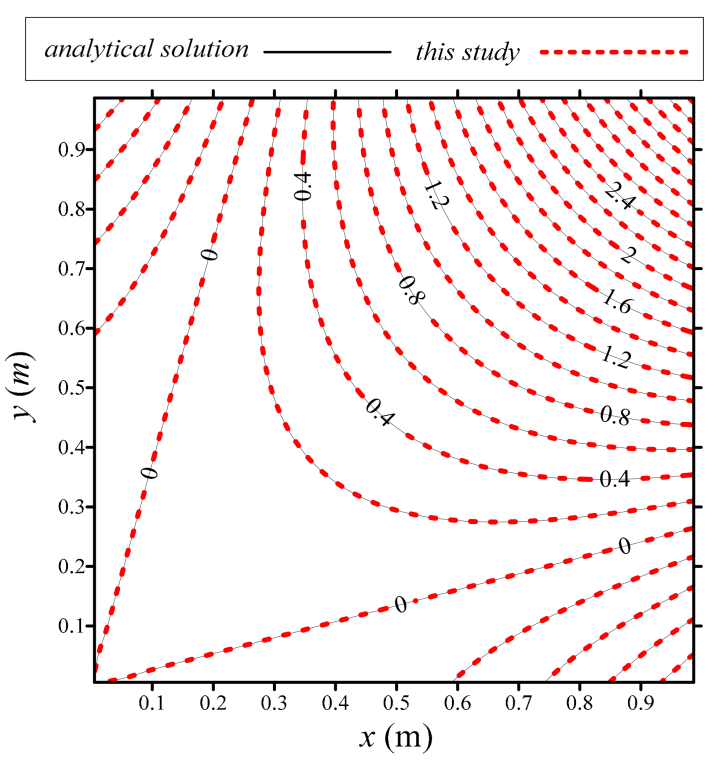

Figure 17. Comparison between the numerical solution and the exact solution. 


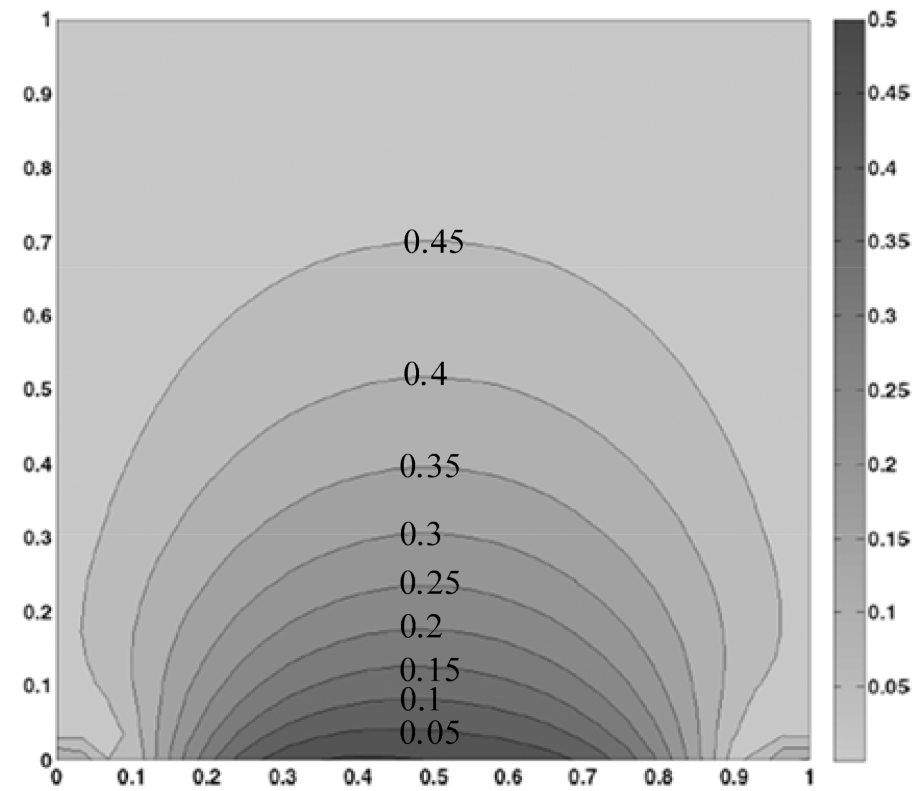

(a)

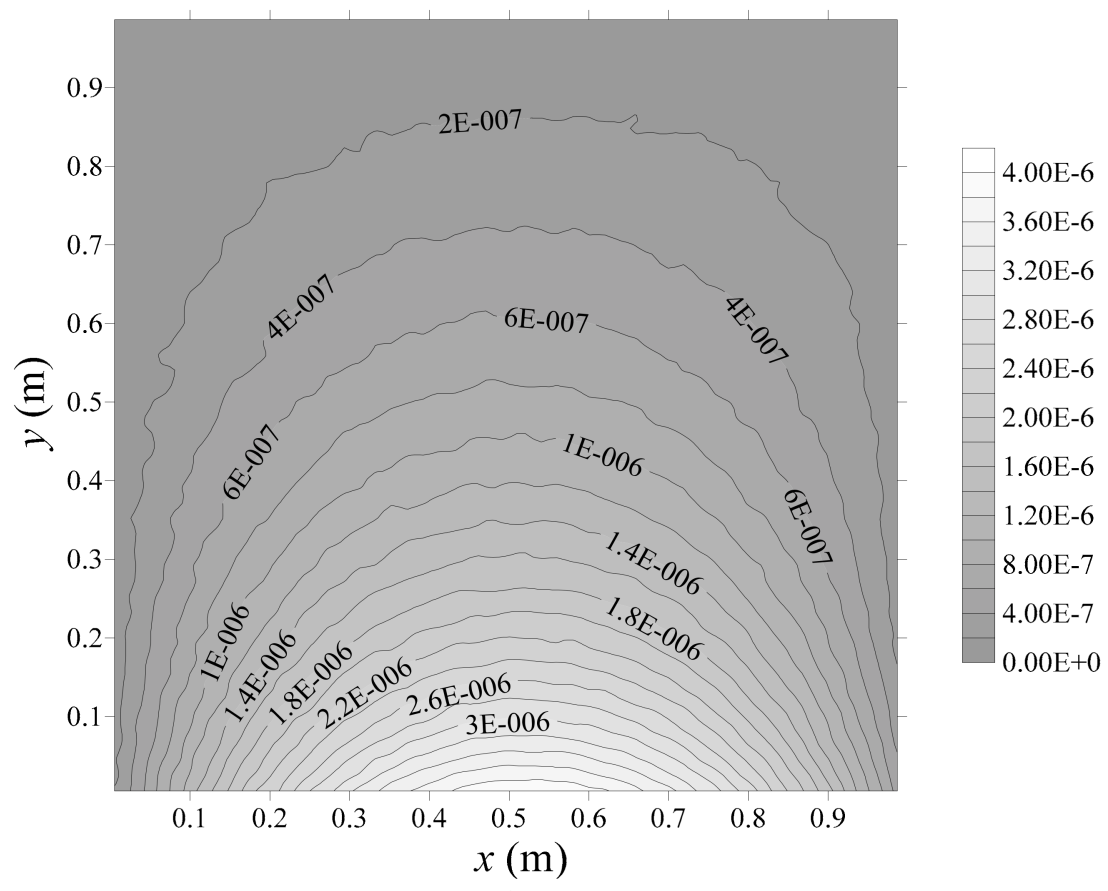

(b)

Figure 18. Absolute error of example 4. (a) Weighted RKCM, (b) Proposed approach.

Table 2. The MAE by assigning different levels of noise for example 4.

\begin{tabular}{cc}
\hline Noise Level & This Study \\
\hline$s=0$ & $3.96 \times 10^{-6}$ \\
$s=1$ & $4.10 \times 10^{-3}$ \\
$s=2$ & $8.90 \times 10^{-3}$ \\
\hline
\end{tabular}




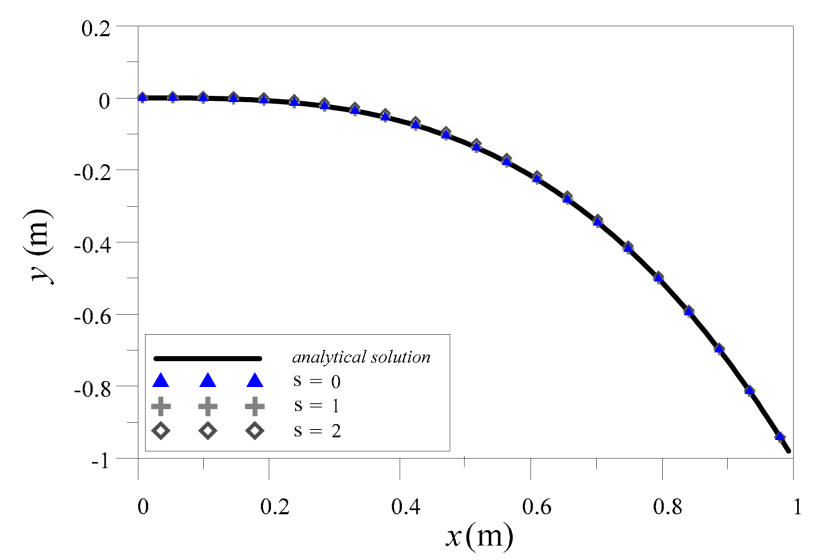

Figure 19. Comparing of the numerical solutions of the proposed method and the exact solution along $\Gamma_{1}$.

\subsection{Example 5}

We investigate a two-dimensional inverse heat conduction problem in a doubly-connected domain, as depicted in Figure 20. An annular region has an outer radius and inner radius of $3 \mathrm{~m}$ and $1 \mathrm{~m}$, respectively. To evaluate the accuracy of the MSMM, we compare the computed results of the method with those of the GFDM [34]. The two-dimensional object boundary is described by the following equation:

$$
\begin{aligned}
& \Gamma_{1}=\left\{(x, y) \mid x=\rho_{1} \cos \theta_{1}, \quad y=\rho_{1} \sin \theta_{1}\right\}, \quad \rho_{1}=3 \quad \mathrm{~m} \\
& \Gamma_{2}=\left\{(x, y) \mid x=\rho_{2} \cos \theta_{2}, \quad y=\rho_{2} \sin \theta_{2}\right\}, \quad \rho_{2}=1 \quad \mathrm{~m}
\end{aligned}
$$

where the radial coordinate is denoted by $\rho_{i}$ and the angular coordinate is denoted by $\theta_{i}$. The exact solution is given by:

$$
T=-\frac{1}{2} x+2 y+\frac{1}{4}\left(x^{2}-y^{2}\right)
$$

$N_{q}$ is the Trefftz order of the basis functions and is set as 15. For solving this example, as depicted in Figure 20, the boundary data are imposed on the inner boundaries $\Gamma_{3}$ and $\Gamma_{7}$. Moreover, there is no information on the outer boundaries $\Gamma_{1}$ and $\Gamma_{5}$. Figure 21 shows the collocation points on the physical domain. The DDM [32] is adopted to solve this problem. At the interface, continuity in flux and temperature must be achieved. We split the problem into two simply connected sub-domains, as depicted in Figure 21. For each sub-domain, 300 collocation points are collocated on the domain uniformly. The sub-domain $\Omega_{1}$ includes $\Gamma_{1}, \Gamma_{2}, \Gamma_{3}$, and $\Gamma_{4}$ and the sub-domain $\Omega_{2}$ includes $\Gamma_{5}, \Gamma_{6}, \Gamma_{7}$, and $\Gamma_{8}$. At the interface, $\Gamma_{2}, \Gamma_{4}, \Gamma_{6}$, and $\Gamma_{8}$ should achieve continuity in temperature and flux conditions. Therefore, the over-specified boundary conditions are given as follows:

$$
\left.T\right|_{\Gamma_{2}}=\left.T\right|_{\Gamma_{6}},\left.\quad \frac{\partial T}{\partial n}\right|_{\Gamma_{2}}=\left.\left.\frac{\partial T}{\partial n}\right|_{\Gamma_{6}}{ }^{\prime} \quad T\right|_{\Gamma_{4}}=\left.T\right|_{\Gamma_{8}},\left.\quad \frac{\partial T}{\partial n}\right|_{\Gamma_{4}}=\left.\frac{\partial T}{\partial n}\right|_{\Gamma_{8}} .
$$

To evaluate the stability of the MSMM, different noise levels of $s=0$ and $s=3$ are considered in the numerical model. To examine the accuracy, we collocate 200 inner points to obtain the temperature field in each sub-domain. The GFDM is also adopted to solve the same problem. The MAE by assigning different levels of noise for example 5 is depicted in Table 3. It can be seen that the absolute error is relatively small, and should be noted that the accuracy of the numerical solution decreases with the increasing noise level. The computed results demonstrate that the accuracy of the proposed method can reach to the order of $10^{-1}$ while $s=3$ is considered. Moreover, the numerical results demonstrate that the accuracy of the MSMM is superior to that of the GFDM, as depicted in Figure 22. 


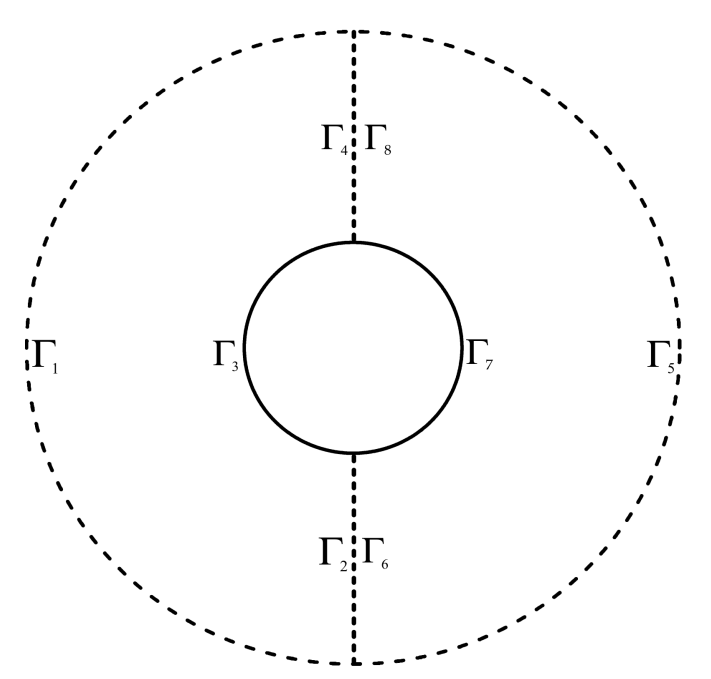

Figure 20. Configuration of sub-boundaries.

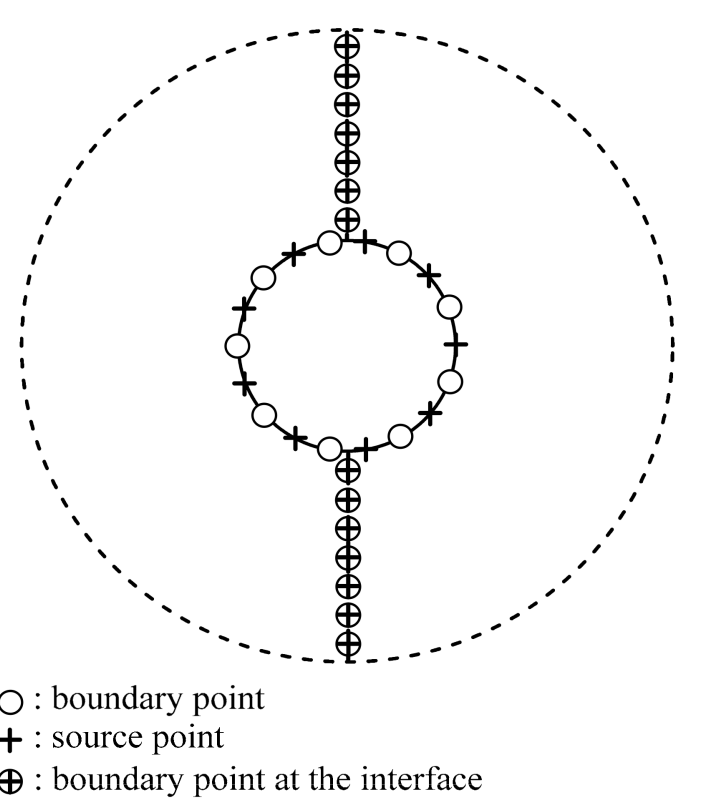

Figure 21. Boundary and source points.

Table 3. The MAE by assigning different levels of noise for example 5.

\begin{tabular}{ccc}
\hline Noise Level & The GFDM & This Study \\
\hline$s=0$ & $7.77 \times 10^{-12}$ & $2.79 \times 10^{-11}$ \\
$s=3$ & $5.08 \times 10^{-1}$ & $3.37 \times 10^{-1}$ \\
\hline
\end{tabular}




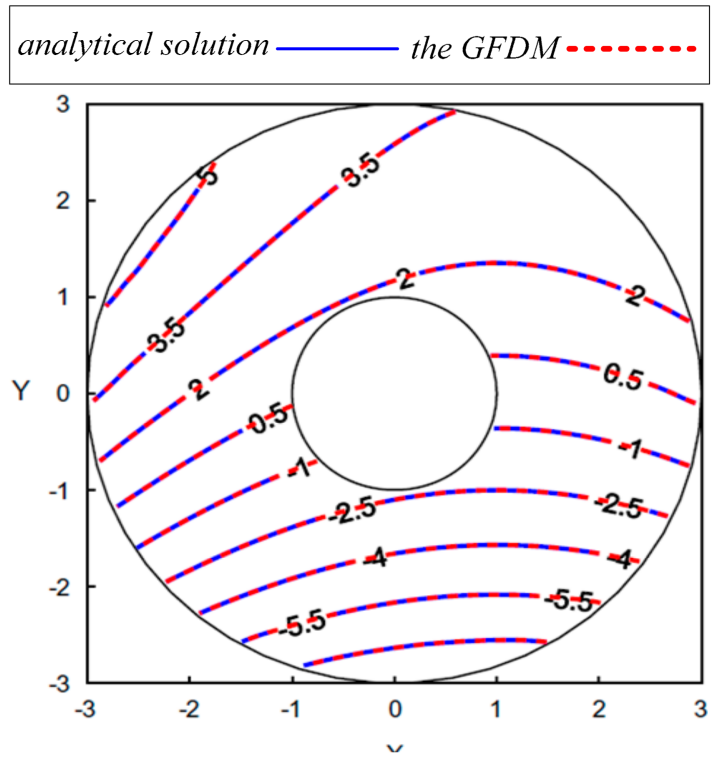

(a) The GFDM

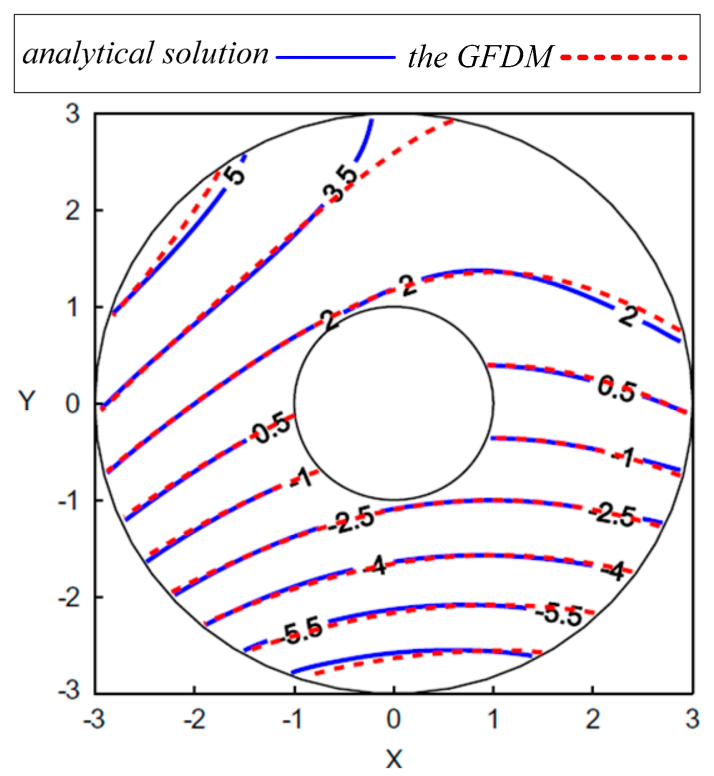

(c) The GFDM

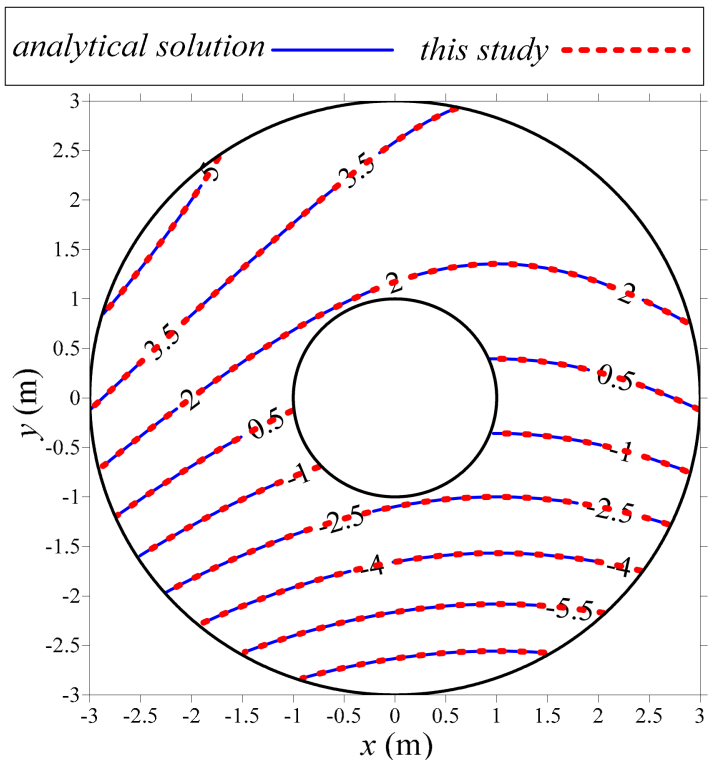

(b) This study

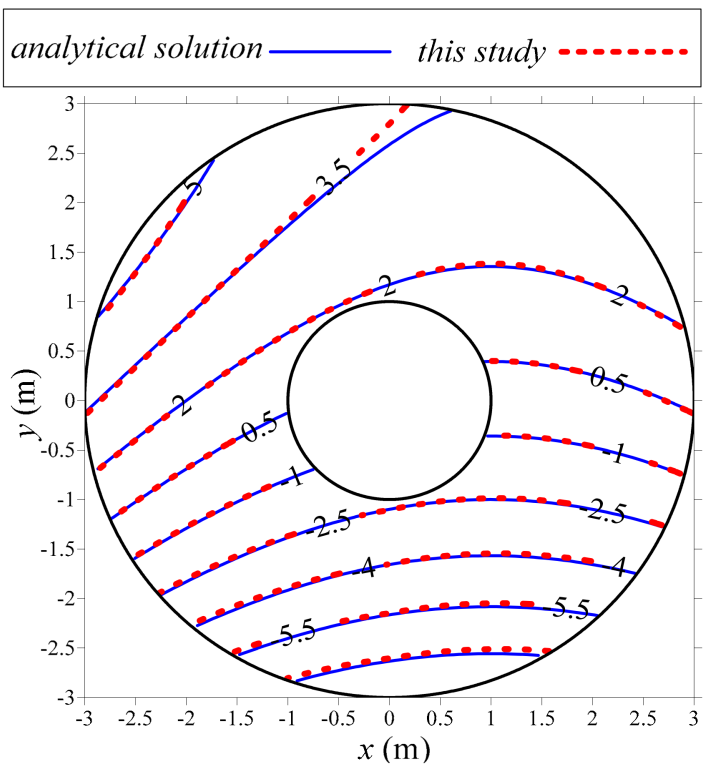

(d) This study

Figure 22. Comparison between the temperature fields of the proposed method and the generalized finite difference method. (a,b) the computed results without the noise and the exact solution and $(\mathbf{c}, \mathbf{d})$ the computed results with a noise level of $s=3$ and the exact solution.

\section{Discussion}

Inverse problems are remarkably challenging because of their ill-posed phenomenon. Although mesh-based numerical approaches combined with numerical techniques, such as the Tikhonov's regularization technique and the L-curve method, have been widely used to mitigate the ill-posed phenomenon of inverse problems, shortcomings in terms of stability and accuracy still exist. In this study, a newly developed MSMM that is capable of solving inverse heat conduction problems in two dimensions is presented. To the best of the authors' knowledge, the pioneering work using the proposed MSMM to solve the inverse heat conduction problems has not been reported in previous studies yet. The contributions and limitations of the proposed MSMM are discussed below. 
This study first adopts the MSMM to solve the inverse heat conduction problem. The MSMM is a highly accurate numerical method because specific solutions are adopted. Non-singular basis functions are derived from the general solutions of the governing equation and provide a highly accurate numerical solution. The method is especially advantageous for solving inverse problems because it can mitigate the ill-posed phenomenon and obtain results with higher accuracy. Moreover, regularization techniques are not required for solving the inverse problems. The MSMM maintains the same superiorities of the CTM, such as the boundary discretization only, and is advantageous for solving inverse problems.

However, limitations still exist. For example, the MSMM approximates the solution using many source points by the addition theorem, it can only be applied for linear governing equations. Five examples are carried out in this study. Result comparisons with previous published data are also conducted. The results from Examples 1-3 demonstrate that the MSMM may obtain better accuracy than the MFS. In addition, the results from Examples 4 and 5 depict that the MSMM is more accurate than the weighted RKCM and the GFDM. Finally, the results from all examples show that the proposed method may recover the unknown data with very high accuracy.

\section{Conclusions}

In this study, we present a newly developed MSMM for solving the two-dimensional inverse heat conduction problems. Several numerical examples are provided to validate the accuracy of the method. To the best of the author's knowledge, this may be the first study to adopt a new type of a meshless method such as the MSMM to solve the inverse heat conduction problem. The conclusions are summarized as follows.

1. The MSMM boasts the same advantages of the CTM such as using only boundary discretization only and demonstrating efficacy for solving inverse problems. The proposed method does not require mesh generation, numerical quadrature, or any regularization techniques for solving inverse problems. Moreover, we successfully adopt the DDM for solving the inverse heat conduction problem bounded by a doubly-connected domain.

2. Numerical examples are presented using the proposed method. The results are compared with those of other meshless approaches, such as the MFS in conjunction with the ECSHA, the weighted RKCM, and the GFDM. The comparison of results demonstrates that the proposed method is superior to other meshless approaches, especially in terms of accuracy.

3. Finally, the results demonstrate that the proposed method may recover unknown data with remarkably high accuracy, although the over-specified boundary data are assigned a portion that is less than $1 / 10$ of the overall domain boundary.

Author Contributions: This study was conceptualized by C.-Y.K. Data curation was conducted by W.-P.H., W.Y., and C.-Y.L. The methodology was presented by J.-E.X., and the validation of the method was performed by J.-E.X. and C.-Y.K. wrote the original draft of the manuscript, and C.-Y.K. and J.-E.X. modified the document after review and revision.

Funding: This study was partially supported by the Ministry of Science and Technology of R.O.C. under project grant number MOST 108-2119-M-019-001.

Acknowledgments: The authors thank the Ministry of Science and Technology for providing financial support.

Conflicts of Interest: The authors declare no conflict of interest.

\section{References}

1. Marin, L. A meshless method for solving the cauchy problem in three-dimensional elastostatics. Comput. Math. Appl. 2005, 50, 73-92. [CrossRef]

2. Marin, L.; Elliott, L.; Heggs, P.-J.; Ingham, D.-B.; Lesnic, D.; Wen, X. An alternating iterative algorithm for the Cauchy problem associated to the Helmholtz equation. Comput. Methods Appl. Mech. Eng. 2003, 192, 709-722. [CrossRef] 
3. Hon, Y.-C.; Wei, T. A fundamental solution method for inverse heat conduction problem. Eng. Anal. Bound. Elem. 2004, 28, 489-495. [CrossRef]

4. Mera, N.-S.; Elliott, L.; Ingham, D.-B.; Lesnic, D. A comparison of different regularization methods for a Cauchy problem in anisotropic heat conduction. Int. J. Numer. Methods H. 2003, 13, 528-546. [CrossRef]

5. Chan, H.-F.; Fan, C.-M.; Yeih, W. Solution of inverse boundary optimization problem by Trefftz method and exponentially convergent scalar homotopy algorithm. CMC Comput. Mater. Continua 2011, 24, 125-142.

6. Ressing, H.; Gadala, M.-S. A practical investigation to solving the inverse problem of crack identification through vibration measurements. Eng. Comput. 2003, 23, 32-56. [CrossRef]

7. Gilberta, R.; Hackl, K.; Xu, Y. Inverse problem for wave propagation in a perturbed layered half-space. Math. Comput. Model. 2007, 45, 21-33. [CrossRef]

8. Qian, Z.; Fu, C.-L.; Li, Z.-P. Two regularization methods for a Cauchy problem for the Laplace equation. J. Math. Anal. Appl. 2008, 338, 479-489. [CrossRef]

9. Gu, Y.; Wang, L.; Chen, W.; Zhang, C.; He, X. Application of the meshless generalized finite difference method to inverse heat source problems. Int. J. Heat Mass Transf. 2017, 108, 721-729. [CrossRef]

10. Yeih, W.; Chan, I.-Y.; Ku, C.-Y.; Fan, C.-M. Solving the inverse Cauchy problem of the Laplace equation using the method of fundamental solutions and the exponentially convergent scalar homotopy algorithm (ECSHA). J. Mar. Sci. Technol. 2015, 23, 162-171.

11. Bourgeois, L. A mixed formulation of quasi-reversibility to solve the Cauchy problem for Laplace's equation. Inverse Probl. 2005, 21, 1087-1104. [CrossRef]

12. Andrieux, S.; Baranger, T.-N.; Abda, A.-B. Solving Cauchy problems by minimizing an energy-like functional. Inverse Probl. 2006, 22, 115-133. [CrossRef]

13. Wang, J.-G.; Wei, T.; Zhou, Y.-B. Tikhonov regularization method for a backward problem for the time-fractional diffusion equation. Appl. Math. Model. 2013, 37, 8518-8532. [CrossRef]

14. Yang, F.; Fu, C.-L. The method of simplified Tikhonov regularization for dealing with the inverse time-dependent heat source problem. Comput. Math. Appl. 2010, 60, 1228-1236. [CrossRef]

15. Calvetti, D.; Morigi, S.; Reichel, L.; Sgallari, F. Tikhonov regularization and the L-curve for large discrete ill-posed problems. J. Comput. Appl. Math. 2000, 123, 423-446. [CrossRef]

16. Trujillo, D.-M.; Busby, H.-R. Optimal regularization of the inverse-heat conduction problem using the L-curve. Int. J. Numer. Methods Heat Fluid Flow 1994, 4, 447-452. [CrossRef]

17. Berntsson, F.; Eldén, L. Numerical solution of a Cauchy problem for the Laplace equation. Inverse Probl. 2001, 17, 839-853. [CrossRef]

18. Hào, D.-N.; Giang, L.T.T.; Kabanikhin, S.; Shishlenin, M. A finite difference method for the very weak solution to a Cauchy problem for an elliptic equation. J. Inverse Ill-Posed Probl. 2018, 26, 1-23.

19. Bilotta, A.; Turco, E. A numerical study on the solution of the Cauchy problem in elasticity. Int. J. Solids Struct. 2009, 46, 4451-4477. [CrossRef]

20. Lesnic, D.; Elliott, L.; Ingham, D.-B. A alternating boundary element method for solving cauchy problems for the biharmonic equation. Inverse Probl. Sci. Eng. 1997, 5, 145-168. [CrossRef]

21. Liu, C.-S. A modified collocation Trefftz method for the inverse Cauchy problem of Laplace equation. Eng. Anal. Bound. Elem. 2008, 32, 778-785. [CrossRef]

22. Liu, C.-S.; Qu, W.; Chen, W.; Lin, J. A novel Trefftz method of the inverse Cauchy problem for 3D modified Helmholtz equation. Inverse Probl. Sci. Eng. 2017, 25, 1278-1298. [CrossRef]

23. Young, D.-L.; Tsai, C.-C.; Chen, C.-W.; Fan, C.-M. The method of fundamental solutions and condition number analysis for inverse problems of Laplace equation. Comput. Math. Appl. 2008, 55, 1189-1200. [CrossRef]

24. Jin, B.; Zheng, Y. Boundary knot method for some inverse problems associated with the Helmholtz equation. Int. J. Numer. Methods Eng. 2005, 62, 1636-1651. [CrossRef]

25. Yiqian, H.; Haitian, Y. Solving inverse couple-stress problems via an element-free Galerkin (EFG) method and Gauss-Newton algorithm. Finite Elem. Anal. Des. 2010, 46, 257-264. [CrossRef]

26. Chan, H.-F.; Fan, C.-M. The local radial basis function collocation method for solving two-dimensional inverse Cauchy problems. Numer. Heat Transf. B Fund. 2013, 63, 284-303. [CrossRef]

27. Chang, C.-W. A new meshless method for solving steady-state nonlinear heat conduction problems in arbitrary plane domain. Eng. Anal. Bound. Elem. 2016, 70, 56-71. [CrossRef] 
28. Fu, Z.-J.; Chen, W.; Zhang, C. Boundary particle method for Cauchy inhomogeneous potential problems. Inverse Probl. Sci. Eng. 2012, 20, 189-207. [CrossRef]

29. Xiao, J.-E.; Ku, C.-Y.; Huang, W.-P.; Su, Y.; Tsai, Y.-H. A novel hybrid boundary-type meshless method for solving heat conduction problems in layered materials. Appl. Sci. 2018, 8, 1887. [CrossRef]

30. Ku, C.-Y.; Xiao, J.-E.; Liu, C.-Y.; Fan, C.-M. On modeling subsurface flow using a novel hybrid Trefftz-MFS method. Eng. Anal. Bound. Elem. 2019, 100, 225-236. [CrossRef]

31. Ku, C.-Y.; Xiao, J.-E.; Liu, C.-Y.; Yeih, W. On the accuracy of the collocation Trefftz method for solving twoand three-dimensional heat equations. Numer. Heat Transf. B Fund. 2016, 69, 334-350. [CrossRef]

32. Young, D.-L.; Fan, C.-M.; Tsai, C.-C.; Chen, C.-W. The method of fundamental solutions and domain decomposition method for degenerate seepage flow net problems. J. Chin. Inst. Eng. 2006, 29, 63-73. [CrossRef]

33. Yang, J.-P.; Guan, P.-C.; Fan, C.-M. Weighted reproducing kernel collocation method and error analysis for inverse Cauchy problems. Int. J. Appl. Mech. 2016, 8, 1-21. [CrossRef]

34. Fan, C.-M.; Li, P.-W.; Yeih, W. Generalized finite difference method for solving two-dimensional inverse Cauchy problems. Inverse Probl. Sci. Eng. 2015, 23, 1-12. [CrossRef]

(C) 2019 by the authors. Licensee MDPI, Basel, Switzerland. This article is an open access article distributed under the terms and conditions of the Creative Commons Attribution (CC BY) license (http://creativecommons.org/licenses/by/4.0/). 